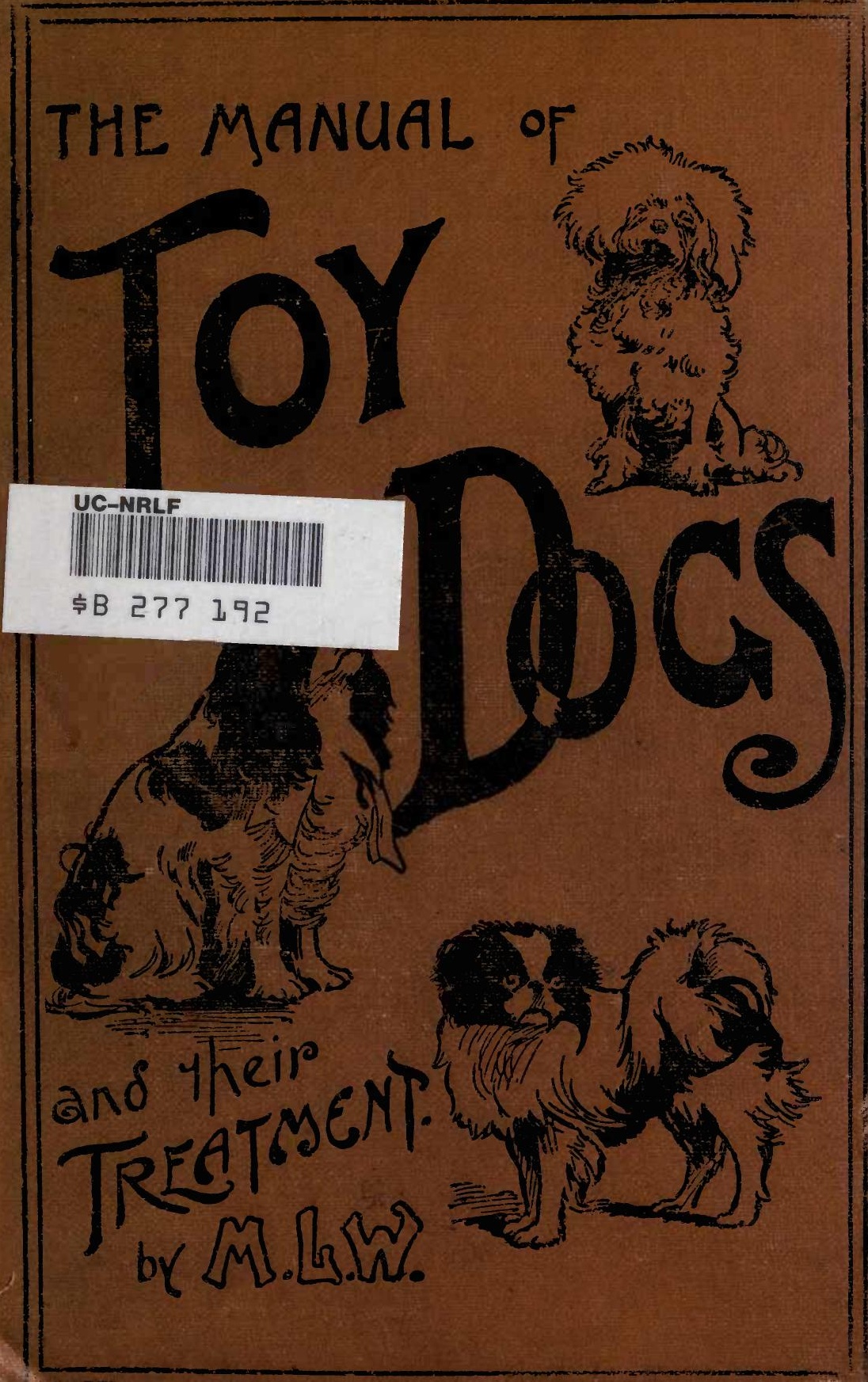



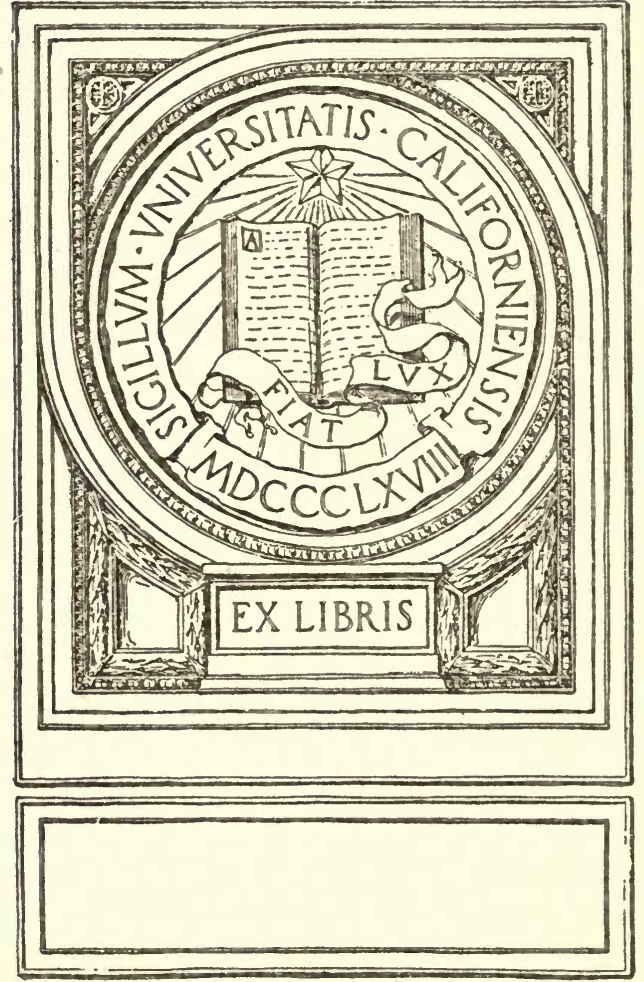




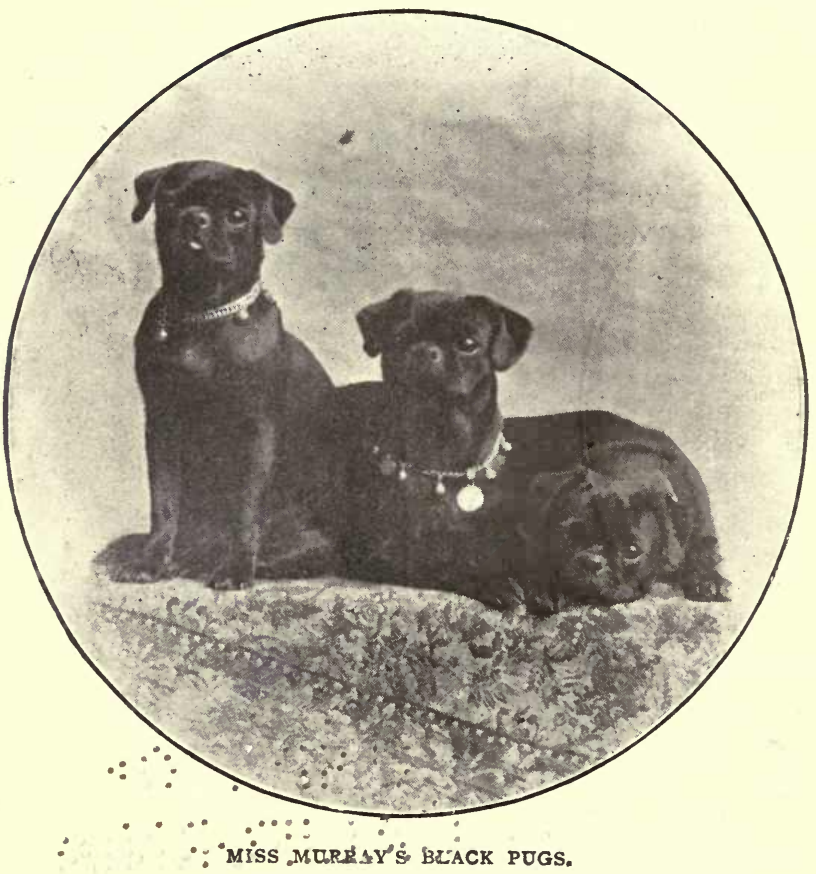

Frontispiet. 


\title{
A MANUAL OF
}

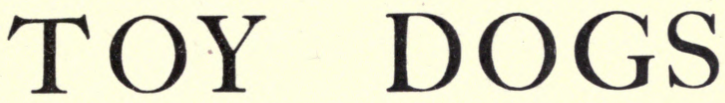

HOW TO BREED, REAR, AND FEED THEM

MRS. LESLIE WILLIAMS

THIRD EDITION

THIRD IMPRESSION

\author{
LONDON \\ EDWARD ARNOLD
}

41 \& 43, MADDOX STREET; BOND STREET, $\boldsymbol{W}$. 


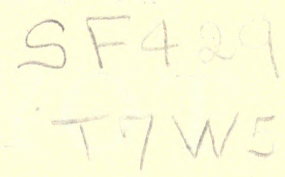

Copyright 1904

- Alll rights reserved

$\because \vdots \vdots \vdots \vdots \vdots \vdots$

$\because \vdots, \because \vdots \ldots \therefore \therefore \because \cdots$ 


\section{PREFACE TO THE THIRD EDITION}

THIs little book, in its earlier editions, met with so uniformly kind and gracious a reception, that I am encouraged to hope it may still make new friends on this, its third appearance. It has given me the greatest pleasure to hear from correspondents in many countries that they have found it as helpful as I hoped a manual drawn entirely from actual personal experience might prove to be.

In the years which have elapsed since I first wrote upon dogs, there has been a wonderful advance in veterinary science and practice. Operative surgery under anæsthetics has become nearly as confident in relieving our pets as in abating our own miseries. Much disease, however, is still present among dogs for which there is no warrant in Nature, and which might be entirely conquered in the course of a few generations, could the prejudice against natural and rational diet be completely abandoned. To persuade dog-owners to give meat-feeding a trial - one honest experiment has never in my experience failed to convince the most sceptical-has been my constant endeavour, and I cannot let the "Toy Dog Manual " go forth on another journey without once more laying emphasis on the fact 
that the really successful dog-owner's secret is a very simple one, spelt in the four letters-MEAT. I have to thank numerous kind friends for help in providing the illustrations, nearly all pictures of actual presentday winning dogs, and examples not only of beauty and show points, but of perfect health. I am also greatly indebted to The Illustrated Kennel Neres for the loan of blocks and for other kind courtesies, as also to The Ladies' Field, a paper devoted in its kennel columns to the best interest of dogs.

\section{L. WILLTAMS.}

Swanswick, Bath,

May $5^{t h}$, rgro. 


\section{CONTENTS .}

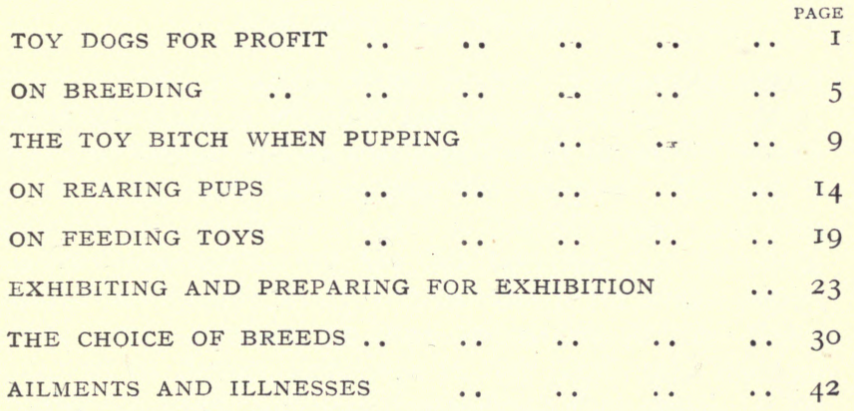

CLUB STANDARDS, DESCRIPTIONS AND POINTS OF VARIOUS TOY BREEDS $\ldots$.. $\quad \ldots \quad \ldots 80$

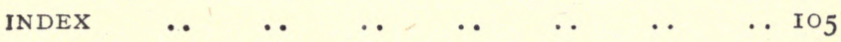





\section{A MANUAL OF TOY DOGS}

\section{CHAPTER I}

TOY DOGS FOR PROFIT

Perhaps the question which is most frequently asked anent toy dogs is whether the keeping them as a pleasure and hobby can be combined with profit by means of breeding them and selling the puppies. To such a query it is very hard to give a definite reply, for this reason-whether or not toy dog breeding can be made profitable depends, firstly, on the character of the enterpriser, and, secondly, on that inscrutable factor-Fate. Some of us devote ourselves to our dogs, take endless trouble for them, and spend money on them freely, with the poorest possible return; others, while not making nearly so much fuss about their pets, manage to turn out healthy litters at regular intervals, and sell them at remunerative prices. All that can be done is to put before the novice " how not to do it," and leave to each individually the chances called luck, for which their star is answerable. Taking one year with another, and presupposing patience, perseverance, affection for the dogs, and some business-like qualities in the aspirant, I am of opinion that toy dogs can be made to pay their expenses, and leave a margin of profit; this in the case of non-exhibitors. Where exhibiting is contemplated, the luck element is still more to the front, and a degree of experience, both local and general, is essential to success. If success, however, in winning prizes is once attained, the sales of puppies become much more assured, and higher prices are naturally obtainable. 
As a means of eking out a small income, dog breeding is occasionally successful, supposing the breeder to possess advaritayes in the way of proper quarters, and plenty of time to spare, natural aptitude not being wanted" buit I should greatly hesitate to suggest to a Foor lacy, without experience in dogs, that she should embark capital in such a venture. Many people seem possessed with the idea that they have only to buy a female dog, or dogs (generally the latter, since the novice is always inclined to split upon the rock of overcrowding and overstocking at first), and get it mated with some well-known sire, to ensure a fine, healthy litter of pups, which can be immediately sold at high prices, having in the meantime been fed on dog biscuit and attended to, more or less, by any one who happens to be at home. No greater mistake! If you want to succeed with toy dogs, you must, at any rate until you have considerable experience and, in addition, the ability to direct others and make them understand, which is never an easy task, look after the pets yourself, not spasmodically, but regularly; see that they have exercise and proper food in proper quantity and variety, and at fixed and regular hours ; you must have an eye always open to notice the smallest beginnings of illness-a watchfulness servants, for example, never can comprehend, still less practise ; and lastly, you must set an aim before you and keep to it with perseverance, even though you may, and probably will, often feel impatient and despairing. Then, too, you must be prepared to nurse the dogs properly if, or when, they are ill. Nobody can expect to be exempt from illness, dog or man, and good nursing is as needful in the one case as in the other. A sick toy dog must be kept clean, petted, sat with, talked to, and tempted with nice things, like a sick baby, for the little spirit has much to do with the tender frame, and pain and weakness need sympathy, and respond to it eagerly. A little toy bitch, accustomed to fly to her owner at every impulse, cannot be left to have puppies all alone-though her fussy preparations, which may last all night, are rather wearisome. 
Some one must stay with her and comfort her until her troubles are over; otherwise, she will fret and worry until, when the pups do appear, she has no milk for them.

All these little requirements and necessities may seem absurd to those who think a dog is a dog and nothing more; but we have bred generation after generation of toys to be in our constant company, and made them almost humanly intelligent, while, naturally, their small brains have no human balance; and that a nervous toy dog does need such consideration will be granted, I am sure, by all successful breeders. At the same time, I am by no means advocating the silly system of overpetting and overfeeding, whereby dogs can be made a nuisance to themselves and every one else. Because a child must be taken care of, it does not follow that it need be spoiled: we ought to put a hat on its head when it goes out in the sun, but we need not walk beside it, holding an umbrella over it ; and so with our small dogs-they must be watched and cared for, but they need not, and should not, be coddled and made silly.

I have no opinion of a dog which will not go out because it is raining, preferring to make itself objectionable in the house; or of one which leaves the small proportion of biscuit in its dinner and comes round scratching your arm for more meat; or of one which rushes back to the fire when a walk is suggested on a chilly day. Dogs like this have not been properly cared for; it is not affection for them, seeking their wellbeing, but downright silliness, which is responsible for their self-indulgent ways. Thanks be that toy dogs of this kind are becoming much less common, and indeed, in the case of any person desiring to keep them with an idea of profit, such ways would be discouraged by self-interest, for pampered dogs are not those which breed freely and do their puppies justice.

Where it is necessary that the dogs shall pay their way, it is of the first necessity that the inevitable expenses of starting and gaining experience shall be carefully considered. It is not a bad plan to get a 
little cheap dog, and see it through a litter before embarking in a "paying" breed, as where these are concerned it is useless to expect return unless a really good price has been paid for valuable stock to begin with. One does occasionally see such toys as Japs and Poms advertised very cheaply; and I have known people who studied these advertisements with rosy visions of "picking up" a bitch from an excellent strain, at a guinea or two-with some slight fault, like a few white hairs, to cheapen her-of breeding show stock from her and making a little fortune. Chances like this seldom come in the way of the novice. The best start a would-be breeder who is without any experience can have, is by placing herself in the hands of some one who has been successful, buying a young bitch which comes of a winning strain, though it may possess some fault, at a fair price-which will not be a small one-and taking the breeder's advice as to mating, etc. Or it is by no means a bad plan to buy a brace of unrelated young puppies and rear them. Of this, more in the chapter on breeding.

To buy imported or pedigreeless small toys for breeding is a complete lottery. Foreign breeders are extremely careless with regard to their strains, and purity of blood can never be depended on. Another point which must be insisted upon in relation to profitable toy breeding is the necessity for health in the kennel. I say kennel because it is a useful word, but am far from suggesting that toys of any kind should be kept in the way understood by " having a kennel " among larger dogs. The breeder who succeeds best is invariably the one who keeps one or two, or even four or five, pet bitches, running about the house enjoying full liberty and all the happiness of personal favourites, with, it may be, a dog also of the party. The breeder who is most troubled with skin complaints, distemper, lengthy vet's bills, and all the expenses, such as sick diet, which eat up profits, is the one who has built or fitted " kennels," no matter at what expense, and filled them with dogs. 


\section{CHAPTER II}

\section{ON BREEDING}

VERY small bitches, and especially those belonging to certain breeds which are known to be "shy," are not only often reluctant to breed at all, but are not infrequently very indifferent mothers, while there are great risks to the bitch in pupping where the sire is larger than herself, or where larger dogs occur in the immediate ancestry on either side. For these reasons, brood bitches are always wisely chosen of medium size, and mated to very tiny dogs. In all the breeds which come under the head of toys, smallness is a desideratum, tut the practice of inbreeding which has been extensively resorted to cannot be too highly condemned; while the equally mistaken idea of attaining this end by underfeeding puppies has also contributed to the weakliness of constitution which is an immense drawback to some breeds. Reckoning size by weight is another faulty practice much against the true interests of toys, which we want to be small and healthy at the same time; for a very tiny dog, if compact and sturdy, may weigh much more than a leggy specimen which, to the eye, seems half as large again.

A bitch from 5 lbs. to 7 lbs., if, as I said before, of a small strain, may be safely used for breeding, and the smaller the dog the better, provided he is healthy. The plan of sending away bitches to a stud dog saves the expense of buying a dog of one's own ; the sire's wins help to sell the puppies very materially, and the good offices of his owner may generally be reckoned upon to assist the novice; but there are other facets to the question.

These tiny dogs, which are frequently exhibited, are often very unreliable sires; they work too hard, and their owners are sometimes very indifferent as to whether the visiting bitches are satisfactorily attended 
to. True, the terms always do, or certainly always should, include a second visit free if the first proves fruitless, but there is the loss of time, the disappointment to the owner, and sometimes to the little bitch herself, who may have been quite anxious to breed and not have had a fair chance, and the trouble and expense of travelling for her. On the whole, I am much inclined to advise the novice to, at any rate, begin by rearing up a male puppy of such breeds as Pekingese and Griffons, or the scarcer toy Bulldogs, and using it for the home stud ; for the other plan is less likely to result in disappointment when a little knowledge has been gained of the kennel world in general. This, of course, unless the whole thing is gone into under the ægis of some experienced owner, as before suggested. Some little bitches are exceedingly capricious, and will not take the least notice of a strange dog, where they would willingly mate with one they knew and liked; others are so upset by a journey and a strange place as to be useless pro tem. ; others, again, instead of being ready to breed twice a year, as is the usual habit of female dogs, may only come in season once in twelve months, and then but fugitively. In such cases it is a positive necessity to have a dog on the spot. Where a sire must be chosen from among strangers, his points should correct any in which the bitch is deficient ; your toy pug may have too small a head, with little wrinkle-you must look for a dog with good head properties as her mate; your Pom may be long in back, and you must seek a male with the opposite quality, and a plume well over and touching his frill.

The first puppies of two young dogs are generally larger than the parents, but I do not believe the theory often advanced that the first litter is always the best. Puppies by a very old sire are usually small.

A toy bitch, if sent away, should be carefully packed in a roomy, warm basket; the provision of draughty, tumble-to-pieces baskets is false economy, both for show and breeding purposes. If possible, a toy dog of 
either sex should have a cosy little basket kennel, with a door, which it can use at home as a sleeping-place, and in which it can travel; the basket can be fitted with an outer case of wood for greater security, but the dog will stand the journey much better if it is in a familiar basket. Something with a peaked or rounded top should be chosen; the ventilation being safer in this, as flat-sided and flat-topped packages may be so

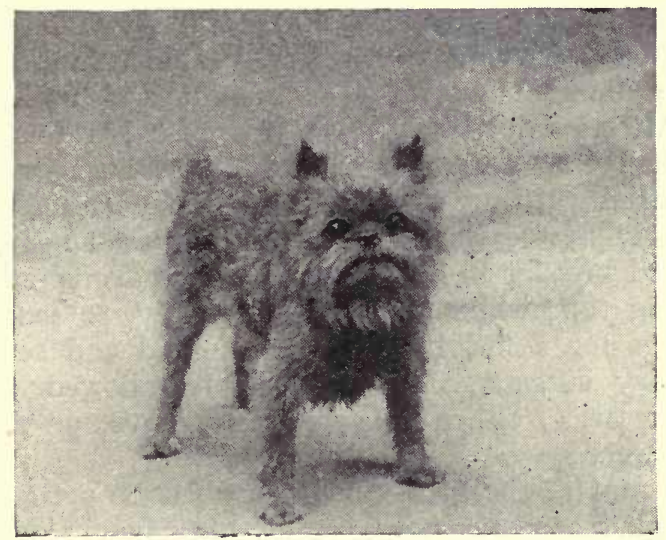

GRIFFON BRUXELLOIS.

"Sparklets," the property of Miss Fohnson.

crowded upon with others in a guard's van as to suffocate the inmate.

The usual period of willingness to breed in a toy bitch is, more or less, one week. This is preceded by about a fortnight's preparation, a week or so of gradual enlargement of the parts concerned, and a week of a coloured discharge from the uterus and vagina. Either or all of the stages may last a longer or shorter time ; but three weeks is generally accepted as the period. No attempt at mating the bitch should be made during the first two stages; it is when the discharge begins to cease that she is ready, and the correct judging of this 
time is what chiefly puzzles amateurs, though after they have once been through it they will not find any difficulty. As a rule, bitches are sent away too soon, and as the conveniences for keeping them at the stud dog's house are often few, they are cooped up for day after day, and may become quite "stale" and dull before the real mating time comes-a poor prospect. If the two dogs are in the house together, the male should be kept entirely away from the female from the very beginning of her attraction for him, until she is ready, otherwise he will worry her incessantly and become himself ultimately indifferent and useless in the matter. Toy dogs should never be left to themselves in breeding matters; it is highly dangerous to do so, especially if they are young and inexperienced, and I strongly advise the beginner either to get some experienced breeder to overlook matters and give advice, or failing this, when the female is ready, to send the two dogs for a few hours to some kind and sensible veterinary surgeon. They should be allowed to be together twice, either on consecutive days, or with a day between.

Once mated, the little toy bitch must be petted and taken good care of : not overfed, but given plenty of good, nourishing food, and systematically exercised. If she is in pup it will become evident about the fifth to the seventh week. Some dogs show it much more than others; whether she has puppies or not, she will have the natural provision of milk for them. If she does not pup, she may very likely come in season again in half the usual time. A failure to prove in pup is generally evidenced by a time of great heaviness and dulness, the bitch sleeping a great deal, getting very fat, and decidedly stupid; under these circumstances give her extra exercise and one or two small doses of sulphate of magnesia in food, to ward off skin irritation, a not uncommon correlative. People are far too apt to decide that "missing" is the bitch's fault; certainly she is apt to miss if she is too fat at the time of mating, 
and Nature often, and very sensibly, arranges that she shall do so when she has been regularly bred from at her seasons for a number of times; but outside these occasions it is quite as often the dog's fault as not.

A question which is frequently asked is as to the desirability or otherwise of giving a toy bitch worm medicine, or an aperient, while she is in pup or just before her babies arrive. It is as well to give one mild dose of worm medicine about the end of the third week, if the bitch is known to be troubled with these parasites to any great extent; but it would be much better to have dosed her before her breeding time came on. As to the aperient before pupping which we often see advised, it is a totally unnecessary interference with Nature, and when castor oil, a violent irritant to dogs, is employed, it is a sheer piece of cruelty, likely to have very bad effects.

\section{CHAPTER III}

\section{THE TOY BITCH WHEN PUPPING}

Too much interference is generally alternated in the case of dogs with a disregard of their natural feelings where the arrival of puppies is concerned. It is quite natural that the little bitch, feeling distressed and uneasy, should claim a great deal of notice and attention, and if she has been made a pet of she will expect, and deserve, to be allowed to have her puppies in her mistress's dressing-room or some similar luxury; in which she should be indulged. But once she has got over the preliminaries, which I will presently describe, she should, if possible, be left to herself as far as manual assistance goes. Nature will bring the puppies into the world far better than our clumsy hands, and the merest little tyro of a year-old bitch generally possesses the marvellous instinct teaching her to put her babies 
comfortably afloat on the sea of life. The disregard of a pet dog's feelings at which I have hinted may take the form of sending a tiny bitch out to the stable to pup under the care of a coachman or groom, and this may or may not be cruel according to whether she has any affection for the man or any knowledge of her temporary quarters; personally, I should consider it an unkind thing to do under any circumstances.

The beginning of the toy bitch's trouble is apparent to her owner almost as soon as to herself. She pants, and runs about excitedly, scratching here and there, making wildly impossible and absurd nests for her puppies in all kinds of unsuitable places. This may last for days, but is generally only done for a few hours before the puppies arrive, which, by the way, will be nine weeks after mating. Some bitches shriek in a very distressing way before they pup, and, as a rule, food is refused, and the little mother that is to be is often sick. No anxiety, however, need be felt. As soon as she really means business she will quiet down and settle in the place prepared for her, which by choice should be a big, deep arm-chair, with a white blanket - any old thing will do that is clean-folded in the seat of it, and over this an old cotton sheet, likewise folded, and so secured that the bitch cannot scrabble it up in the foolish endeavour to improve human bed-making which always possesses dogs, and, if indulged, lands them in desperate discomfort on the top of a kind of volcano of rags!

In nine cases out of ten a bitch chooses to pup in the night, and the hours often seem very long, while she may lie and sleep in evident uneasiness, getting up every now and then to make her bed, and panting as if exhausted. It is quite safe to leave her in this condition for twelve hours, but if by that time she seems to be getting weaker and no puppies have come, the vet's services should be requisitioned. Probably she will not eat, but she may be offered a little cold milk. On no account give ber anything hot, externally or inter- 
nally, and do not be tempted to do anything whatever to her; the only interference which is ever excusable is the application of a very little sweet oil or vaseline externally, which she will lick off, and which does no harm and no good, in my experience.

If help is called for at all, it must be the skilled aid of a surgeon ; any other is worse than useless.

The puppies are born singly, and if a bitch has a

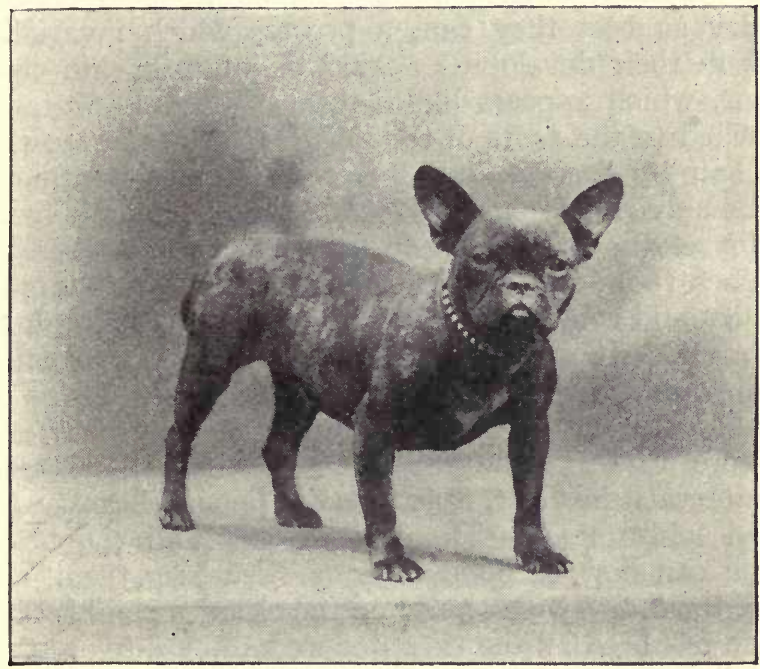

FRENCH TOY BULLDOG.

"La Reine des Roses," owned by Mrs. Townsend Green.

large litter they generally come in twos and threes, with a very short interval between the items of each brace or trio, and a long rest between the batches. The first services the mother has to render her babies are to free them from the bag of membranes in which they are born, and to bite the cord which joins each puppy to the afterbirth-a fleshy substance which comes away with or shortly after it. All animals intensely dislike being watched while they perform these opera- 
tions ; but every bitch who is anything at all of a mother will manage them perfectly. Next comes the licking of the puppies, which have been enclosed each in its membranous bag full of liquid (the liquor amnice), and are consequently dripping wet. Here is the crucial test: a good mother licks her babies until they are warm and dry, then feeds them, and snuggles down with them into a contented heap of intense happiness. A bad mother, on the contrary, leaves her poor infants to dry as best they can, a process which invariably ends in their developing a kind of infantile skin complaint, which appears like a scab of cheesy substance attached to the roots of the hair. It grows away with the hair by degrees, and gets well without treatment, but is ugly and disfiguring for the time being, and a sad evidence of incompetence on the part of the mother.

When the family have settled down, and the puppies are dry and comfortable, it is time to give them a little attention. Have a saucer full of nice, warm milkgruel, made with patent groats as daintily as for an invalid, and let the mother drink it, which she will be sure to do with gratitude; she may have more at intervals during the first day. Then roll away the soiled folds of sheet from under her and the litter, which can now be done without disturbing them, and leave them cosily ensconced on the clean, warm blanket, which has been all the time underneath.

A little later the mother may be put out into the garden for a few minutes, not more than two or three; but she must not be allowed to get chilled. After the first day she should go out for a little walk morning and afternoon, the time of her absence to be gradually lengthened as the puppies grow older.

Until they begin to crawl, valuable toy puppies are much safer and better upstairs in a big chair as described, or in a flat basket with a folded blanket at the bottom set upon the chair, than they can possibly be in any stable or in the kitchen premises, for, no matter how warm, such places are draughty too. There is abso- 
lutely nothing about a litter of little toys, if healthy, to be in the least offensive anywhere, and a good mother will keep them in the very pink of perfection for nearly a month under such circumstances.

Where a poor or weakly mother is concerned, and where the puppies are restless, squall, and seem damp and comfortless, it is another matter. By constant attention as to the changing of the bed, partial handfeeding from a small old silver spoon with cream and hot water, and Plasmon or Lactol, half and half (better than milk, though warm milk will do), and a great deal of patience, the mother may be helped out and the puppies saved; but where they are not valuable it is better to destroy all but one or two ; and where they are so, a good foster-mother offers them by far the best chance of life and health. There are people who make it their business to supply fosters, and one of these should be applied to as soon as possible ; taking pains to ensure, by careful examination on arrival, that the stranger has no skin disease and is free from objectionable insects.

Small toy bitches sometimes have but little milk at first, but by giving warm food only for the first few days, and plenty of milk to drink, it generally comes all right, and so long as the pups seem fairly content, all is well; the flow is sure to increase. Both before and after pupping there is generally a little diarrhœa, which is of no consequence; but if it goes on beyond the second day after pupping, get the bitch on to her usual diet, with a little cold milk to drink, and stop all sloppy foods. Oatmeal, as gruel or otherwise, should never be given after the second day. A discharge, of mucus mixed with blood, is usual after pupping, and may continue for several weeks in gradually lessening amount. 


\section{CHAPTER IV}

\section{ON REARING PUPS}

AN indispensable adjunct in the rearing of valuable toy puppies, which, as a general rule, do far better in the house than in any stable or out-of-door premises, is one of Spratt's or Boulton and Paul's little houses and runs. As personal and vicarious experiences are all that any writer can adduce to support theory, I may be allowed to describe the procedure which has been found successful with my own puppies-born, bred, and reared in house and garden as they are.

Directly they leave the basket of their infancy (in which, par parenthése, I must say, I think them more delightful, helpless little soft morsels, than even when they begin to run about, show intelligence, and need feeding) they are introduced to one of these useful abodes, comprising a sleeping house, provided with a cosy blanket, freely washable and often changed, and a little wired-in run about $4 \mathrm{ft}$. by $2 \mathrm{ft}$. The bigger this the better, of course; and if it has a floor, as some have, pierced with small holes and draining into a removable tray to be kept full of earth, or sawdust, it will be well. Mine is a humbler affair, floorless, and stands on a piece of oilcloth, covered with a large sheet of brown paper, which can be daily renewed; yet it answers its purpose very well. In this, with outings two or three times a day, for variety, the puppies live until they are seven weeks old; the mother, loose about the house, visiting them at her inclination and sleeping with them. At between three and four weeks old they must be taught to lap, which is easy enough with some pups and difficult with others. Warm, boiled milk should be the only addition to what the mother gives them until they are over a month old : it is a mistake to hurry puppies on to patent foods, bread and milk, and the like. Do not let them have a saucer and upset 
it, tumbling into it and getting themselves in a mess, to dry all sour and disagreeable, but hold their little heads one by one as they lap, for they will nod into the saucer and send the milk flying.

As soon as the puppies are strong on their legs, they need more exercise and fun than the run can allow them, and now is the time to take them off the carpets, which they will never respect in after life if they have been allowed to treat them evilly as elderly babies. It is not a bad plan to let them live in the kitchen from this time forth, various things being provisional. One is, that the presiding genius will see to their little meals under your supervision; - that is, you feed them four times a day, and she or he undertakes to see that no one else does so. Another, that the kitchen opens into the, or a, garden, and that the puppies can run there in the sunshine, in warm weather, and so insensibly learn manners ; yet another, that it is a warm, draughtless place, with a nice corner for their sleeping basket. Some folks, whose lower regions do not answer this description, or whose servants are not amenable, may have an occupied stable at command, where the puppies can have a loose box or stall. This plan I do not recommend, for toy pups do far better in constant human companionship; but it, or the alternative one of keeping them in a room with an oilcloth floor, are all that offer themselves, failing the desirable kitchen. I have known toy pups do splendidly in a sunny little room, floored with cork carpet, provided with cosy sleeping boxes, and opening into a terrace-walk, where on all fine and sunny days they were allowed to play; but they were not too much left to themselves, and their apartment was carefully looked after, and brush and sawdust-pan kept going, just as, in my kitchen, the servants hasten to remove any unbecoming traces of their presence. This period, while toy pups are too young to be trained, too old for their mother to clean them up, and also so young as to require warmth and constant watching, is the troublesome one in their live's 
and the one in which so many of them die. Neglect, or dirty surroundings, are fatal to these little delicate atoms, which really call for the same attention we should give a baby; monotony-being kept shut up in one small room for hours or days-and lack of fresh air, carry off many; while sour milk, meals left about in odds and ends, irregular feeding, and lying to sleep in draughts, are all elements of danger. We want to give them warmth and dryness, without stuffiness and overheating; we want to give them sweet, tempting, clean little meals, regularly, four times a day, just as much as they can eat eagerly and no more; we want to give them a cosy day-bed to go to sleep whenever they feel inclined-which will be often-and, lastly, to let them have all the fresh air and out-of-door sunshine they can get without fear of chill. Thus it is that summer puppies, born in the spring, with all the best weather before them, do so much better than those which have the critical teething period to pass through in winter time.

A toy puppy grows more quickly than, for instance, a terrier, and, of course, is adult far sooner than a big dog; the short-haired varieties, again, coming to maturity sooner than the long-coated ones. A Yorkshire terrier is adult at a year, but does not get his full beauty of coat until he is two years old, or thereabouts. A toy Schipperke is, so to speak, grown-up at ten or eleven months, but goes on thickening and improving in shape, and probably increasing and hardening in coat for another year at lest. A Pom's jacket gets grander at each moult until he is three years old. As a general rule it may be laid down that the dog is a puppy no longer at ten months, when his teething is almost always entirely completed. This same teething is a tiresome process, comprising the change of the first set of wee ivories for the permanent forty-two which are to carry the owner through life. Nearly every puppy suffers more or less in the process, some from fits, some from skin irritation, some from colds in the 
head and eyes, some from general feverishness ; but the troubles are ephemeral, and generally subside between whiles, returning as each big tooth is cut. What makes the worst trouble is when the first teeth are severally not shed, but remain in situ, a second tooth forcing itself up at one side of the lingering intruder. This condition is pretty sure to mean teething fits, of which more anon. Dentition begins about the fourth

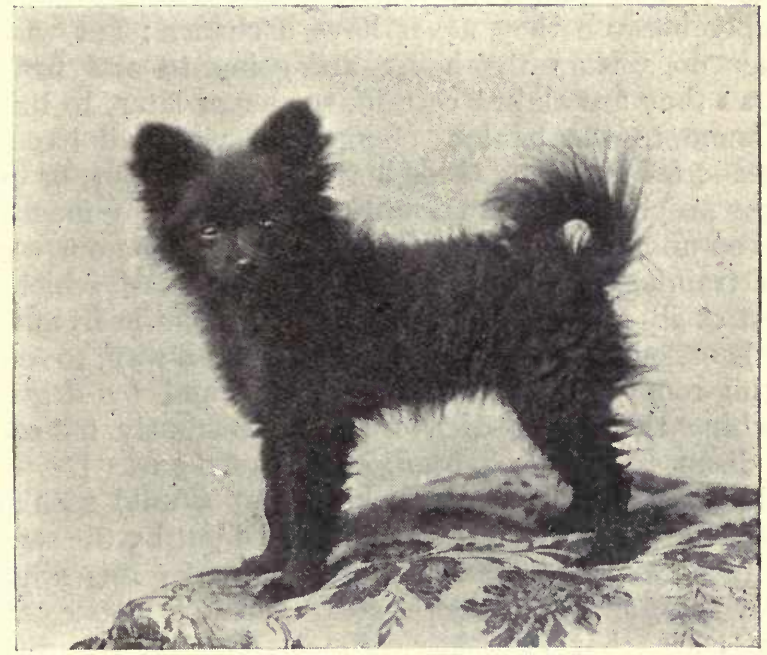

POMERANIAN PUPYY.

At the ugly age.

month, and once safely over, the dog may be considered well reared.

Distemper, that is, the two diseases usually so described, are a bugbear, but it is enough to say that no puppy ought to have them. If he does, it is because . some one has allowed him to get the contagion, by accident or carelessness ; left to himself, he could not indulge in it, for it is not, cannot be, spontaneous.

Small skin troubles, such as puppy pox, in which 
the skin in the under parts of the body is red, and small pustules form and suppurate, after the manner of chicken pox-though puppy pox is not catching-often affect the strongest puppies; and a pup which " teeths with a rash" is generally thought by breeders to be one which, if in the way of contagion, will not take " distemper" very badly, if at all, though whether there is any foundation for this opinion I cannot undertake to say. Personally, my puppies never have distemper, simply because they never have a chance; but where other dogs from the house are going to and fro to shows they are almost certain, sooner or later, to bring it home to the babies. Some day we shall have a crusade for stamping these horrible diseases out, or discover prophylactics, no doubt ; at present they must be looked upon as ill-luck which may never come our way. The training of puppies to the house is a task which is most easily accomplished by bringing them in from the kitchens, or wherever they live in a general way, to some sitting-room for a short time daily, and by degrees teaching them that each offence is instantly followed by dismissal to the garden, or out of doors. Beating little dogs is useless and unkind, but a mild scolding may be given and the infant be carried out by the scruff of its neck. The great thing is to make this sequel invariable, as dogs have a great sense of justice, and soon learn that they have done wrong in this case; whereas, if they are allowed to do a thing three times and beaten for it on the fourth occasion they quite fail to understand the reason of the rebuke.

Some breeds of toys are much easier to teach than others; personally, I have found Poms comparatively difficult dogs to train to the house, and black-and-tan terriers are seldom altogether reliable; while fawn pugs are generally averse to going out of doors in wet or very cold weather ; but patience and perseverance will do it in almost all cases. On the other hand, some little dogs take to the house at once, and give no trouble at all from the very first. A dog just off a journey, or strange to a 
place, is not generally well-behaved just at first, so that the buyer of a puppy, warranted trained, ought to give it a little law before deciding that its education is not properly complete. I am sometimes asked if there is not some magical preparation which cures dogs of untidy habits, but am compelled to own that, in the present state of our knowledge, such a thing not only does not exist, but does not seem likely to be discovered! Small puppies, under three or five months, are physically incapable of resisting any impulse, therefore it is quite useless to attempt to train them too soon. Comparison between the sexes in this matter is sometimes made; some preferring males as house dogs, and others females. I fancy there is not the least difference, and certainly, given a promising and intelligent individual, a little boy pup is as easy to teach manners to as a little girl, and per contra. Much depends upon character; here and there we find some toy dogs which have mean, cringing spirits, and these are generally the ones which won't go out in rain. They may be vulgarly described as " sneaks," and I would not keep a dog of this description. Mere timidity is a different thing altogether, and can be eradicated by kindness and judicious petting. The "sneak" is no companion, and should not be bred from. It will not follow well out of doors, is seldom a good mother, and is apt to transmit its faults of disposition to its offspring.

\section{CHAPTER V}

\section{ON FEEDING TOYS}

In feeding toys, variety is essential, and it is also desirable to give them food which will nourish and support the constitution without fattening them unduly, or heating the blood. It is far better to give a toy a very small dinner, as far as bulk is concerned, of roast meat cut up; or a little boiled mutton and rice; or a bit of 
cutlet minced, than to give a much larger dinner of rice and biscuit flooded with milk or soup. Big, sloppy meals are most undesirable, and the last meal at night, above all, should be dry. Half a penny sponge cake makes an excellent supper for a toy dog, or a couple of Osborne biscuits. Toy dogs should never be given any biscuit containing oatmeal or Indian corn meal, or peameal. These two are much used in dog-biscuit making, on account of their cheapness, and they are both too heating for toy dogs, and, in quantity, indigestible, although oatmeal is occasionally valuable, as in the form of groats, to be made into milk gruel and given to bitches after confinement. Rice, well boiled, is used as a staple, to give bulk to meals, by all breeders of Yorkshire terriers, and it is a valuable food, for this purpose, for it does not fatten, and is as easily digested as any cereal can be. Although I advocate small, dry meals as against large, sloppy ones, I do not mean to say that a certain amount of bulk is not desirable-it is, for without it there would not be the natural stimulus of distension to the intestinal canal. But although the dog has a very large gullet and can swallow, and wishes to swallow, very large quantities as compared to its size, its stomach is not so very large in proportion, and the juste milieuenough and not too much-is easy to ascertain. Eating between meals is quite as bad for dogs as for babies. They should be fed regularly, and restrained from picking up bits out of doors - which may be poisoned, and are sure to be unwholesome. Many dogs have a shocking habit of scavenging, which often means that they are anæmic and harbour worms; if a tonic and worm dose does not mend matters, a muzzle will.

A toy dog of $5 \mathrm{lbs}$. or $6 \mathrm{lbs}$., which has a biscuit at breakfast time, a varied and tempting meal of meat or fish at lunch, and a piece of stale sponge cake in the evening, is being reasonably fed, and should have a healthy appetite. It is a mistake to feed only once a day, as such treatment is only suitable for dogs so far in a state of nature that they can gorge themselves to 
their fullest and sleep for hours afterwards; and then take hard exercise.

It is quite a modern theory that the sins formerly laid to the charge of meat are all unproven, but it is a perfectly just one. Not only do skin complaints arise from malnutrition, or from improper feeding, or a too large amount of starchy food, but a cure for them is frequently found in changing the diet to one of raw or underdone meat only. This is modern veterinary practice, as set forth by the cleverest man of the dayMr. Sewell-and others whose ability is unquestioned; in the olden times the vet's invariable dictum, whether he understood the case or not-and generally he was in dense ignorance as to whether mange, eczema, or erythema was the trouble-was "No meat!" This idea, like others primarily due to ignorance, dies hard, and the are still to be found people who, ignoring the way a dog's teeth are formed, pronounce his proper diet to be farinaceous, notwithstanding the fact that he was created among the carnivora. Of course, we cannot keep a house pet, altered by centuries of evolution, just as Nature kept him, on raw flesh-for one thing, because he is not living the same sort of life; but the conditions are not so different as to have turned a flesh-eating animal into a graminivorous one.

I write, as I feel, strongly on this subject ; for many a time have I been vexed to see how obstinacy in compelling a dog to live on utterly unnatural food, has made a miserable creature of one that would have been happy, properly fed; and the same applies to many a litter of puppies.

It has long been a common habit to feed puppies on sloppy, farinaceous food, even up to the time when they are well on in getting their permanent teeth; if this is a mistake with larger dogs, it is a grievous folly with toys. People feed their pups four or five times a day on watery bread and milk, Indian corn meal and oatmeal, and powdered biscuit, all slopped with milk; they may even leave it about all day. Some of the puppies, the greedy 
ones to wit, nearly burst themselves, whereupon Nature rebels and relieves the pressure by means of diarrhœa; others, dainty feeders, are sickened after one or two doses, and can hardly be got to feed at all. They loathe their food, and getting them on is a constant worry; presently they begin to be often sick (this is the stomach's protest against being constantly distended with liquid food) and if they have, as most puppies have, the ova of worms inside them, these are immensely encouraged to develop, and lose no time in doing so. A nice preparation for the critical period of teething!

If those who find toy puppies difficult to rear thus, would forsake slops and feed them rationally, they would, I think, share the success of a number of breeders, whose toys are noted for their health and beauty, and whose methods I rely upon to back up my contention. Up to the time the puppy can use its first teeth, give it nothing but milk, pure, sweet, fresh, and warn mixed with plasmon or any other good dried milk powder; cold milk will give the baby colic. Teach it to lap from a saucer of warm milk; either good cow's milk, if you can rely on getting it free from boracic acid; pure cream and hot water to the thickness of milk; goat's milk, best of all ; or, in the last resource, condensed milk, thinned with hot water.

The latter must be the kind which is not oversweetened, and not the kind which has had the cream separated. Up to six weeks I find my puppies do best on milk only; when their little teeth are through, and their mother forsakes them, get them on to solids. A puppy loves to gnaw a lump of stalish sponge cake, or suck a rusk; it comforts him to use his sharp little needlepoints-feeds and amuses him at once. Let him then have milk for breakfast and tea; an Osborne biscuit broken up, a rusk of the kind known as " tops and bottoms," just softened with a little drop of milk, not made into a slop, or a bit of sponge cake, for his dinner and supper. At four weeks he may have a little minced chicken or boiled fish for dinner, or shredded boiled 
mutton; at two months he may be fed like his elders, but with no big lumps of meat. All meat given to puppies should be cut up finely, until they are six months old. As to bones, a big bone is good for a puppy to suck and gnaw; but he must not have any kind of bone which he can swallow in whole or part. For grown-up toys any bones, but those of chicken, game, and fish, are a permissible treat, one at a time, and that time at least a week from the next or the last.

\section{CHAPTER VI}

EXHIBITING AND PREPARING FOR EXHIBITION.

Althougin the profits to be obtained from exhibiting are of a secondary nature, and relative simply to the influence exercised on sales and the way in which showing them brings dogs into public notice, it is well worth the while of the dog owner who has a really good little toy to exhibit it sometimes for the fun of the thing. At a show one can learn more about breeds and points, and all the little details which interest doggy folk, than is possible otherwise ; compare notes with other owners, and obtain many useful hints. I am sorry to say that we can also see a good deal going on which would be well suppressed, and get glimpses of the less attractive side of human nature which keen competition and rivalry are apt to call forth, and which the socialistic mixture of all classes composing "the dog fancy" encourages. "Faking"-dyeing pale tan bright, pulling out coat, or tweaking white hairs, dusting disguising powder into the stained jackets of white dogs, training ears to fall or stand erect (temporarily) in the desired way, with other little improvements, such as clipping the hair from the edges of Poms' ears and from their paws and legs, are all practices nobody would own to, but which nevertheless exist; while even perfectly honest owners are able to bring their dogs 
to the front by legitimate methods which are unknown to the novice, and which she can learn from the initiated. As to the " cruelty" of showing, which Ouida so strongly deprecates, a word may be said. It is certainly not kind to send a little petted toy, accustomed to regular ways and the constant society of its owners to a show " on its own," unattended, and with no care but such as the show officials may feel disposed to bestow upon itoften of a perfunctory character. On the other hand, if its owner takes it to the show, establishes it in its pen, visits it from time to time, feeds it, and takes it out of the show at evening time to spend the night with her, as can always be arranged, I fail to see the slightest cruelty in the matter-in fact, many dogs enjoy being exhibited, and it is quite the exception to see a melancholy face in the rows of pens devoted to the well-caredfor toy section.

The first thing to be thought of where exhibiting is contemplated is getting the dog, or dogs, up to their very best form. A toy which is properly looked after at home ought to be always, more or less, in show condition, that is, as far as Nature's arrangements for the shedding of coat, etc., permit ; but a little extra care for a few weeks before a show is desirable. Short-coated dogs, which, par parenthèse, should never be washed at all if it can be helped, must certainly not be washed for at least a fortnight beforehand, but the least possible trace of vaseline or cocoa-nut oil may be applied to their jackets and polished off with a clean handkerchief ; while brushing and hand-rubbing the right way of the hair get up a beautiful gloss and sheen upon their coat, and a little milk to drink daily helps this effect. Eyes should be washed, and if noses are, as some, unfortunately, are too prone to be, dry, a little vaseline well rubbed in with the finger twice a day will remedy the defect.

Long-coated dogs, of course, need much more attention. They must have extra combing and brushing, and, if dirty or flat in coat, but not otherwise, 
should receive a tub about forty-eight hours before appearing in the ring. For this, use soft, warm water, with, in the case of Poms, whose jackets ought to stand out well, a teaspoonful of powdered borax and a quarter of an ounce of dissolved gelatine to each two quarts of water. The soap used should be carefully chosen, and of the best-Vinolia or E. Cook \& Son's Toilet Soap for choice; common soaps are most unsuitable. Many people also use and much like this firm's Improved Dog Soap. These stiff, stand-out coats are encouraged by habitually brushing the wrong way of the hair, and this is advisable, too, for the manes of Schipperkes. Flatcoated dogs, like Yorkshires and toy spaniels, often spend their lives, the former especially, in the intervals of shows, like summer fire-irons, " in grease "- that is, their coats saturated with oil. To such an extent as this, the preparation may be left to the professional exhibitor (with whom, it is as well to remark, few inexperienced amateurs have much chance, as far as the Yorkshire terrier is concerned); but a little cocoa-nut oil, with the merest trace of cantharides, well rubbed into the roots of the hair for some weeks beforehand, encourages the coat to look its best. Great care is needful in washing white dogs, and only the best of soap should be used; also soft water, with a little borax in it, and a squeeze of a blue-bag in the rinsing-water, to prevent the hair from showing a yellow tinge. Yorkshire terriers must not be rubbed up and about anyhow in their bath; neither must Maltese nor toy spaniels; the hair so carefully kept parted down the middle of the back in the two former breeds must be sponged downwards from the parting, while hot towels and warmed, soft brushes should be used for drying, in such a way as to preserve the habit of growth, which is such a point in these dogs. Rubbing "all over" also encourages curliness-a fatal fault in the breeds mentioned-and this is an additional reason for care. In washing dogs great pains should be taken to dry the insides of the ears thoroughly, and the bath, which 
most dogs so detest, will be robbed of half its terrors if the head is not soaped or soused ; it can be effectually washed with a sponge, thus avoiding the miseries of soap in nose and eyes. Washing, however, as an habitual thing, is most injurious to coat and skin, ruins the colour of black dogs, and should never be made a practice. Daily grooming with brush and comb will keep any properly-fed dog perfectly sweet and clean.

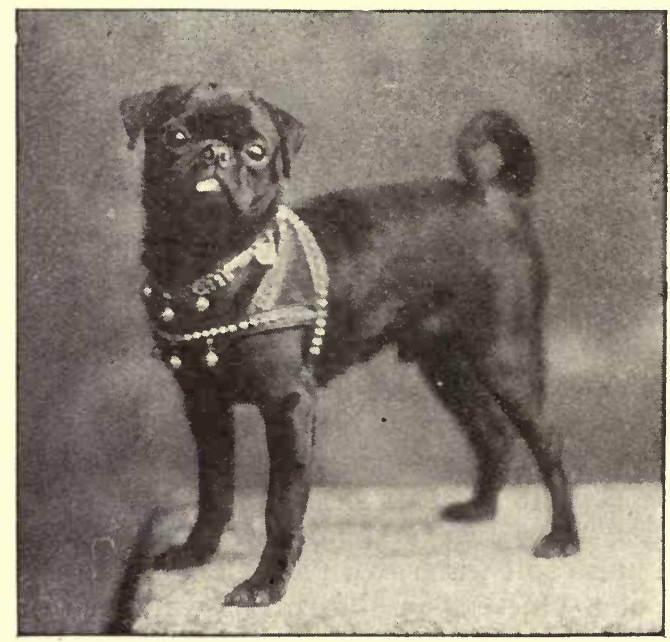

BLACK PUG.

" Fiji," owned by Miss Hyde.

Poodles are, perhaps, as troublesome to prepare for show as any dogs. There are, as yet, no corded toy poodles to speak of, but the curly toys are very delightful little dogs, deserving much more than their present popularity. Their shaving or clipping is, of course, an ever-recurring task, which must at no time be neglected, and is necessary once a month ; but, after the first time or two, it is not at all difficult to manage. The shaved parts should be gone over, the dog having been washed 
the day before, with one of Spratt's Patent Poodle Clippers, a little machine exactly like a small horseclipper, always working against the trend of the hair from the tail along the back to the middle of the body, and from the feet upwards. A pair of scissors, with curved-up points, will be needed for the face and toes, which are the most troublesome parts to do ; but actual shaving with a razor is only done as a finishing touch just before a show. It makes the skin rather tender and is the one part of the toilet, not needful for everyday attire, which calls for expert aid. After clipping, the skin should be well rubbed with a very little white vaseline oil, which brings up a nice gloss and prevents the dog from taking cold. There are various professional poodle clippers in London, among them a lady, who will visit dogs at their own homes for the modest charge of five shillings; but country exhibitors are generally obliged to resort to home talent for the operation.

The long hair is now fashionably arranged in a fluff, teased out with a comb, and well brushed until it stands out; the forelock is tied up on the top of the head with a big satin bow, and voilà, la toilette de monsieur est fini! - the indispensable bracelet and smart collar being alone wanting.

Entering dogs for a show is a simple enough matter. Having ascertained what show you intend to patronise, send a card to the secretary, whose address will be found with the advertisements of the show in the doggy papers, asking for a schedule. On receiving it, read the rules carefully, and also the matter relating to specials, and enter the dog according to the form enclosed; if the show is held under Kennel Club rules, exhibits must first be registered with that body. If merely under Kennel Club licence, this is unnecessary. Occasionally, the reply to, or acknowledgment of, such registration, which is made on a form always sent with schedules and stud entry forms, and accompanied by an indispensable half-crown, is so much delayed that the novice-exhibitor trembles with fear lest her exhibit should be disqualified; 
but such terrors are groundless-so long as the entry has been sent in before the date of the show, all will be well.

The next question is the burning one of escort. Personally I should not like to send little toy dogs to a show without some trusted attendant, and I cannot, therefore, advise anyone else to do otherwise.

Taking them oneself, with maid or man in reserve to leave in charge, is the most pleasant way, for all parties, of arranging matters, and the paraphernalia accompanying is somewhat as follows :-

A warm and comfortable travelling basket for each dogpreferably a little house in which it can sleep at night.

A campstool for the attendant. Standing about at shows is killing work, and chairs are not always obtainable.

Coats for the dogs if the weather is at all cold, for exhibition buildings are almost invariably draughty. The Petanelle coats (sold by Spratt's), of French pattern, with storm collars, are specially warm and smart, and are also aseptic, and the Petanelle cushions are charming in every way.

Some suitable food. Toy dogs will seldom eat what the show authorities provide, and are often too excited to take anything but what is specially dainty. A lunch-basket tin of small pieces of chicken or meat, ready cut up, with the dog's own little plate, will be found useful. Milk at shows is not always reliable, and if any is wanted it should be taken in a bottle, especially for litters.

A brush and comb. A warm, large shawl. I say nothing about the millinery with which people often hang their pens, the satin cushions, etc., with which I can but say the dogs are often made to look extremely silly, but unless there is any rule in the schedule to the contrary, exhibitors are at liberty to provide anything which appeals to their taste in this line. The shawl, or blanket, is often useful for draping round wire pens to keep away draughts, and as such things cannot be got without much trouble once the show has begun, it is as well to be provided beforehand.

Taking dogs out of the show at night can always be managed, usually on payment of a deposit; and the trouble is quite worth while, for fatal colds are apt to be the result of leaving delicate toys to shift for themselves in the colder hours of dark and dawn. 
Leading into the ring is, of course, the crux of the exhibitor's anxiety, for now comes the critical moment -will the dog show or not? Some dogs are born showers-brisk up, look smart and knowing, accept the judge's overtures graciously, and generally exhibit themselves to the best advantage. Others are variable, and cannot be depended upon; will sometimes show well, and at other times-if they are a little out of sorts, for instance, or do not like the look of their rivals in the ring-will not do themselves justice. Others, again, obstinately, lower tail and ears, crouch and cringe, or, worst of all, roll over on their backs. If a dog, after several attempts at showing him, persists in such conduct, it is generally best to give him up as far as exhibition is concerned. But a good deal may be done beforehand to teach little dogs how to show themselves. They may be made accustomed to being led about in a chain, and encouraged to strain from the collar after a ball, etc. Also, they should be taught to receive attention from strangers affably.

Just one word as to the exhibitor's own conduct in the ring may not be amiss. Sometimes old hands at showing are by no means polite to new-comers, sad to say, and will very probably endeavour to screen the novice, if good enough to be a rival, from the judge's eye, by thrusting themselves and their exhibits forward ; while, terrible to relate, such incidents as a sly poke with the foot, administered to a rival's shy dog, or the intentional treading on a toe, are not altogether unheard of. The novice should keep her dog well to the fore, disregard what other exhibitors are saying or doing, so far as strict politeness and good feeling allow, and, while not obtruding her exhibit on the judge's eye, try to get him to notice it in all legitimate ways.

Speaking to a judge in the ring, and while acting, is a great breach of etiquette, unless some question is asked by him, which should be replied to audibly; but most judges are quite willing to give reasons for their decision, or a candid opinion, if asked to do so when 
the judging is over. It is, of course, needless to warn gentlewomen against any show of feeling at being overlooked, etc. ; but the fact that lamentable exhibitions of disappointment do occasionally take place is one not to be denied, while, of course, strict justice is occasionally lacking. Still, taking things for all in all, a very little experience will enable the novice to take her proper place in the show world, where she will be sure to meet with much kindness and unselfish help - such, at least, is my experience; while exhibiting adds a zest to dog owning unobtainable by any other means.

The principal shows where toy dogs are catered for are the Kennel Club Show, in October; the Toy Dog Shows and Cruft's, generally held in February, at the Agricultural Hall; with the shows arranged by the Ladies' Kennel Association, the best of which, from a toy owner's point of view, usually takes place in the summer, and with the provincial fixtures, such as Birmingham, Manchester, and Bristol, and numerous licence shows in all parts of the country, at all of which there is generally a fair classification for toys. All shows may be found advertised in the Illustrated Kennel News and other dog papers.

\section{CHAPTER VII}

THE CHOICE OF BREEDS

THF choice of a breed to take up is generally dictated by personal preference, and fashion has a large spoke in the wheel. Just at present, the fashionable breeds among toys are certainly Pomeranians, or Spitz toyscommonly known as "Poms," Japanese spaniels, Pekingese or Chinese spaniels_-sometimes called Chinese pugs, toy bulldogs, and Griffons Bruxellois. Of the choice of a breed for profit I have spoken before, and will now consider the question from the point of view 
of a lonely dame seeking a pet, or pets, and having no preconceived prejudices.

The Pom, then, is a little dog, hard to get good, but really valuable when so secured. A good toy Pom means one as small as possible, certainly under $8 \mathrm{lbs}$, and preferably under 6 lbs., not long-legged and weedy, but short-backed and compact; with tiny erect ears, a fine-pointed muzzle, small dark eyes, tail-or plume, as it should be called-well over the exact median line of the back; small, fine, and delicate legs and feet, covered with short hair ; and last, but far from least, a profuse coat standing out well all over the body, and amplified about the neck with the characteristic frill, and at the backs of the hind legs with the crinière. Bright brown and chocolate are very much more common than they were a year or two ago, when either was scarce and much desired, but blacks are always favourites. Black-pointed sables (wolf-coloured Poms) seldom have good stiff coats, and, like the beautiful orange sables, are apt to be flat-coated, thus are not so popular ; while parti-coloured dogs depend for attraction upon their quality otherwise. Blues, which, unless large, generally have hairless ears, are very charming, and carry excellent coats, but are comparatively seldom seen. The usual faults of toy Poms are "apple-headedness "term which explains itself - scarcity of coat, coarseness in head or leg, tails badly carried, big ears, or protuberant eyes, legginess and weediness, or curliness. A wave in the coat spoils some from a show point of view, and though washing with borax and water, and combing out with a comb dipped in a weak solution of gelatine, will temporarily remedy the defect, it spoils the desirable bushy look of a Pom to a great extent.

Poms are capital little companions, faithful, exceedingly sharp and intelligent, and generally devoted to one person; they are good with children if brought up with them; but they are fussy and excitable little things, bark a great deal, and have nerves. I do not consider the character some people give them of snap- 
pishness at all justified by facts; but here and there a sharp-tempered Pom may be found. Their quality of disdain towards strangers is one which ought to be considered a virtue in all pet dogs. They are not of the easiest dogs to train to the house, especially when kept in numbers, and are not always reliable in this way, mainly on account of their quick, nervous disposition ; but for cleverness, affection, and beauty, they have few, if any, equals among toy dogs, and they are never likely to lose their popularity; a really good toy Pom is always immensely admired and courted wherever it is taken. Puppies are not now so easily saleable at high prices as was formerly the case, as so many people took them up that they have become plentiful: and it is not worth while to breed second-raters; but a good Pom will still sell.

Next to toy Poms I will mention toy Schipperkes, because, though they are not as yet so fashionable, and probably never will be, they resemble Poms in many ways. As house dogs they are eminently desirable, wonderfully clean and well-mannered, and like the Pom in cleverness and fidelity to one person, while they are much hardier and easier to rear and keep in good condition. They are not at all nervous dogs; but wildly full of life and greedy for exercise ; their incessant activity vying with that of the merry little Spitz. They are decidedly " barky" and exceedingly inquisitive, good travellers, and dogs which settle themselves down anywhere, and are content so long as they are with the favourite "human" they specially possess. Schipperkes are extremely heavy dogs for their size, and quite a wee one will weigh four times as much as a Pom which hardly looks smaller. Both breeds require a meat diet and plenty of good food, which they work off by their active ways ; but the bulk of the Schip's meals should be larger. As a rule, Schips are very good-tempered dogs, and, like Poms, sharp followers at heel. They are, however, pugnacious little things, and have only the grand forbearance of bigger dogs 
to thank for the prevention of many a tragedy due to uppish self-assertion. Black is their colour, and taillessness their most intimate quality; sone, we are told, are born tailless, most-are not! Brown and fawn Schips are common enough in Belgium, the home of the race ; and we have now not infrequently classes for them over here; while whites, which arc really fawns, exist, occurring in litters now and then

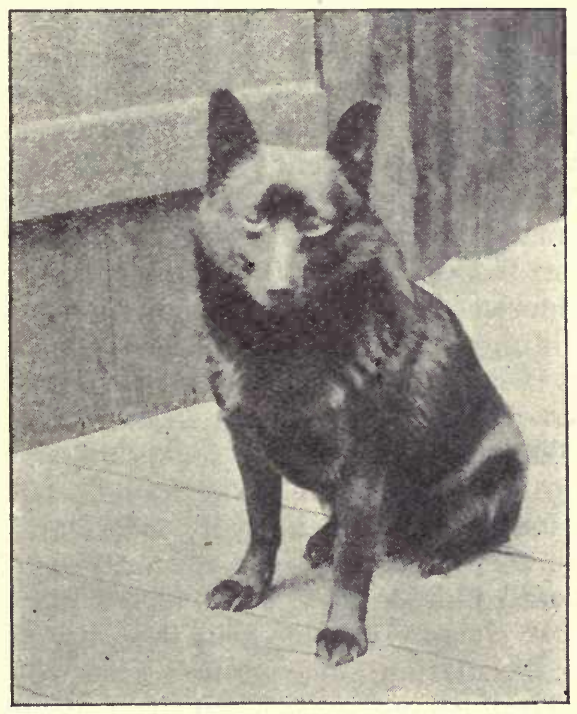

SCHIPPERKE.

"Fandango," owned by Dr. Freeman.

from a throwing back to some distant ancestor, and are really pretty dogs, though I confess the piquancy and charm of the blacks, with their sharply-pricked, thin ears, their rounded-off flank, hard, shiny coats, and dense masses of mane and culotte, the Schip's distinctive points, are to me lost in an " off-coloured" dog. Their faults, as toys, are soft, silky coats, toyish or apple or badly-shaped heads (that universal stumbling block), 
"Pommy," quality of coat (there is no blemish on a Schip's escutcheon greater than a putative cross with a Pom), white hairs or markings, ears which are rounded at the tip instead of pointed, too big, or badly carried, short faces, unlevel jaws, spread feet, crooked or distorted legs, and long backs. The whole appearance of the dog should be very smart and cobby, intensely alert, and altogether clean and well put together, qualities difficult to describe, but which "sautent aux yeux."

Toy bulldogs are yearly becoming more popular. They are absolutely ideal dogs as to temper and all the other qualities necessary for a pet and companion, and almost uncannily intelligent, but alas! they are delicate beyond denying. They are hard to breed, and hard to rear; few of the bitches are good mothers, while their babies have little stamina; they are shy breeders moreover, and altogether need incessant care and watchfulness. If they can have this, well and good, and their puppies will sell immediately ; so that, as a source of profit, they may be recommended, always provided luck and a capacity for taking much welldirected pains are on the owner's side. The prices obtained for these dogs, if really small and of good strain, are somewhat high for the ordinary amateur, while a small bulldog bred from bigger ones, such as can be most cheaply obtained, in the way of a toy, is but a poor speculation, since her first litter will probably kill her. The limit of weight at which a toy bulldog ends and the bulldog proper begins, has been matter of controversy, and the original limit of some $20 \mathrm{lbs}$. was found to present so many difficulties that many breeders desired to have it altered. An equal, or even greater, amount of discussion raged round the question of drop, rose, or bat ears - that is, of upright or falling ones. Finally the sensible decision of having two clubs, one for toys in all respects like the large English bulldogs, and one for dogs of French origin, though now of English breeding, with upright or "bat" ears, to be called 
French toy bulldogs, was arrived at. The English type is now known as the Miniature Bulldog.

Japanese spaniels are quite one of the derniers cris of fashion.* With them I include Rekingese, as al though the latter are hardier dogs altogether, and easier to manage, they are also Eastern, so making things even. Japs are pretty little dogs, of average intelligence and affection, if not quite equal in these respects to the first two breeds discussed. Up to the present " distemper" has been their chief scourge, and keeping them in numbers seems to be an invariable invitation for a visit from some pest, to the contagion of all which they seem peculiarly susceptible. Griffon breeders say that if a Griffon feels ill it dies, and this is in some measure applicable to Japs also. There is no reason why it should be so, for in their native country they are hardy enough, and the cause is traceable to inbreeding, occasioned by the difficulties put in the way of their importation both by the Japanese authorities and our own, and resorted to with the idea of keeping them small; the delicacy caused by the hardships of the voyage, which they stood very badly; to the pioneers of the race over here, and the rush for small sires, often too much used, and over shown. If breeders would buy young, unrelated puppies, feed them on meat, bring them up healthily, and so found fresh strains, this

* Japanese Spaniels. - The five rules of Japanese spaniel beauty, according to the Delhi Morning Post, are these : (I) The butterfly head; (2) the sacred $\mathrm{V}$; (3) the bump of knowledge ; (4) vulture feet; (5) the chrysanthemum tail. To attain the " butterfly head" and the " sacred V," a Jap must own a broad skull with a white $\mathrm{V}$-shape up it (the body of the butterfly), the small, black, V-shaped ears forming the butterfly's wings. The "bump of knowledge" is a small, round, black spot between the ears. The hair on the "vulture feet" feathers to a point in front, but must not widen the slender foot, and to the eye of faith the beautiful, silky, plumed tail, tightly curled over the back, presents the semblance of the national flower, the chrysanthemum. 
delicacy could surely be overcome with comparative ease. In appearance, Japs are extremely fascinating. Their colours are black and white, red and white, and yello: or lemon and white-the latter two combinations being the rarest ; their coloured ears, like butterfly wings, the short-faced head between forming the loly, their heavily fringed feet, and their plumed tail making up a charming and piquant tout ensemble. They are frequently confounded with Pekingese, which are

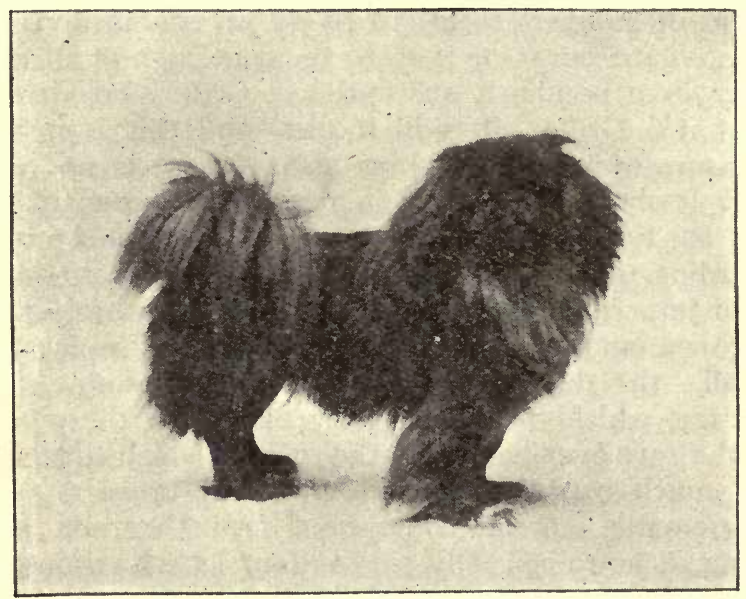

PEKINGESE.

"Foo-Kwai of Newnham," owned by Mrs. W. H. Herbert.

whole coloured, red or yellow, with black markings, and whose ears are not set on at the same angle. A Pekingese pup is perhaps the very prettiest puppy going, before it reaches the lanky stage, which breeders of all toys, except perhaps pugs and Schips, know means the utter indifference, even scorn, of the uninitiated public. The prices of Japs rule fairly high, and a good puppy cannot be obtained, unless by special luck, for less than $£$ IO Ios. ; a larger female pup for a trifle less nerhaps- 
but such, if good in points, are quickly snapped up for brood bitches. Japs have the same toy weight limit as Poms $-8 \mathrm{lbs}$.- - and the over toy weight dogs are far hardier and easier to breed than the midgets.

Griffons Bruxellois are quaintness personified, and their funny little characters, full of dignity and selfsufficiency, are indicated by their no less funny little exteriors. The characteristics of a good Griffon are smallness, hardness of coat, deep, rich red colour, huge black eyes, à fleur de tite, the shortest possible blackended nose, as flat as may be with the face (this appearance generally aided by the breeder, who presses the baby cartilage upwards at every opportunity), and fine and sound legs and feet. The tail is docked, but the ears may not now be interfered with-a righteous rule. An undershot "monkey face" is the desideratum, and though sometimes shy breeders, these little dogs are well worth having, and make the best of house pets.

Of black-and-tan toy terriers there is not much to be said, for the simple reason that they are at present quite out of fashion. A vague idea still, I believe, prevails that the bare and leathery, not to say mangy, appearance some of the former little creatures present about their appleheads and big ears, is a sign of good breeding; indeed, I have often been seriously invited to consider the high claims of a spidery, ill-shaped atom so affected to distinction on the score of aristocratic descent.

In the show-ring things like this are not tolerated, and the really well-bred black-and-tan is not like the little abortions sold-but seldom now, though frequently of old-by itinerant vendors whose characters were far from being above suspicion, and by dog-dealers, as the crime de la crîme of pet dogdom. The show blackand-tan toy is like a miniature Manchester terrierglossy of skin, long and neat in head, with small, dark eyes, oval, not round and goggling; fine, well-made limbs, with the correct pencilling of deep, rich tan on 
the toes. There must be no tan down the backs of the hind legs, and the ears must be neat and well carricd; the tail a whip.

Yorkshire terriers, if small and well coated, always find a sale, and will never be without friends. I like them much as single pet dogs, but a kennel of Yorkshires is a life's work, and only the enthusiast can give

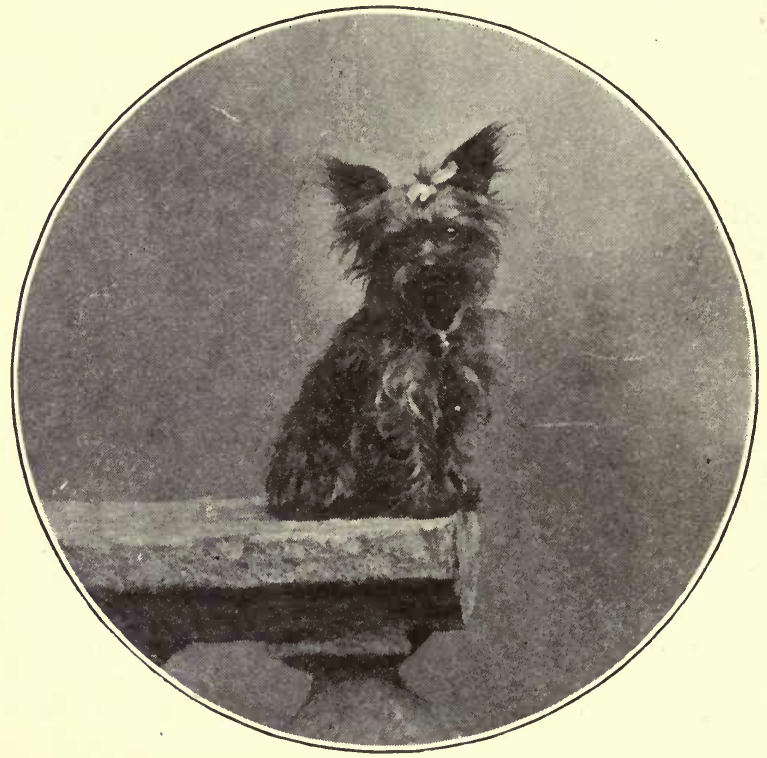

YORKSHIRE TERRIER.

"Trixie," owned by Miss O' Donnell.

them all the care they need. A Yorkie must be brushed (lengthily) every day : it must be rubbed with oils and washes, especially when its hair is breaking, the process which turns the short-coated black-and-tan puppy into the full-blown blue-and-tan beauty of mature age. If the coat is to be done justice to, the puppy must, when ncccssary, be most carefully washed (though washed 
as little as possible), restrained from scratching by having little wash-leather socks kept upon its hind feet, and dieted with every attention directed towards the prevention of any skin disorder. No dog can carry a heavy coat unless well nourished, and the old idea that farinaceous foods sufficed for this is exploded. To avoid anæmia, keep the blood pure and rich, and give strength, a Yorkie must have the nourishment of meat. Withal, it is a merry little soul, and if its coat can be to some extent sacrificed, a good companion, fond of outdoor life, very barky and lively, and tolerably affectionate; but a really lovely show Yorkie is not a being for every day. The breed does not suffer much from " distemper," and, strange to say. in spite of generations of coddling and fussing, and breeding for smallness and coat, is a decidedly healthy one. The white Yorkshires, a new variety some folk have tried to push, is, I think, in no way especially desirable-the Maltese can do all that is necessary in that line; while the attempt to make "silver" Yorkshires popular, too, simply means that bad-coloured dogs without any tan (paleness of tan is the stumblingblock in many a Yorkshire's career), are classed by themselves and offered prizes.

Toy pugs are, I think, invariably fascinating to those who have a liking for pug kind; they are big pugs in little, and everyone knows the points of a pug. My own toy fawn pugs loved their comforts too much to be perfect dogs for companioning a person of active outdoor habits, but they were sweet-tempered, gentle things, and, as such, to be commended. Pugs as a Tace seem strangely apt to skin trouble, and the toys are no exception. I have not seen many really good and very small fawn toys, but there are some, and where a pug is to be bought, a toy is really most desirable. They make good house dogs, and are seldom or never noisy, while those of a comparatively active strain, bred to plenty of outdoor fun, and not indulged in the greediness which, alas! is generally a feature 
in their character, need by no means acquire the stout, snoring wheeziness which some folk think an elderly pug cannot escape. All the same, I can but say that I prefer the black variety on the whole, for they unite the sweet temper, faithfulness, and gentleness of the fawns with an untiring energy, to my mind one of the best qualities a dog can possess. They are also hardier, less subject to " distemper" and kindred ills, and very alert and intelligent. One merit, if such it be, they do not share with the fawns-the latter are not expensive dogs, for thcy are almost always good mothers and prolific breeders. Not that the blacks fail in these respects, but as yet they are comparatively dear-that is, the really good ones. Head properties make much of their value just now, for a good-headed black pug, with a broad skull, large eyes, and plenty of skin and wrinkle, is not in every litter, and narrow skulls are much disliked, though Nature, with characteristic contrariety, seems to rejoice in producing them.

Pugs cannot stand heating foods any more than Yorkshires, which agree with them in doing better upon boiled rice as an addition to meat to make needful bulk, than upon any other farinaceous food. Next to it in value comes wheat meal; oatmeal and Indian corn meal will surely bring skin disaster. Lean meat, underdone for choice, fish, and chicken, may be varied. to make the meals, with a small amount of the needful staple as bu'k.

Toy spaniels in general are not difficult dogs to deal with. They are faithful and extremely affectionate dogs, and the Blenheims make good country pets, having often a considerable amount of sporting instinct, even when they come of stock which has been kept for show only for many years. The Marlborough Blenheims are, of course, examples of the sporting Blenheim, though they are not correct in show points; and there is no reason why one of these dogs, toys though they be, and fit to win, should not be a good little country companion. For towns, white long- 
haired dogs are not to be recommended, because of the occasional washing, which is a vexation alike to dog and owner. The colouring of the Blenheims is very taking, and one with all the show points, spot on the head included, is sure to be admired; but toy spaniels, as a race, the Jap and Pekingese excepted, are very much in the hands of professional exhibitors, and but seldom now seen as pets. The black-and-tan King Charles is inclined to be rather a silly dog, pretty enough, but not "brainy" ; a loving little thing, but unintellectual - such, at least, is my experience of him. The faults of both breeds are generally too much leg, long heads and noses, instead of the big round skulls desired; small eyes, and curliness - the latter a direful mistake. The Prince Charles, or Tricolour, is the King Charles over again in three colours-black, tan, and white; and the Ruby is, as its name implies, all red; rather scarce, this is, to my mind, the prettiest of the toy spaniels. All are very susceptible to damp and cold, and should be carefully dried, especially as to the feet, after being out in rain or mud. They are sweet dogs in skin, and seldom smell " doggy"-a great virtue.

Maltese have a good many friends. These are the oldest of all lap dogs, and a good specimen, with perfectly straight hair-which is, however, but seldom found-is really a thing of beauty. They should be treated like Yorkshire terriers, except that some of the ever-recurring tubs may be avoided by dusting flour or violet powder (pure starch) into the coat and well brushing it out again. They are often spoiled by brown noses, which are a great handicap, and also by the brown marks caused by running of the eyes, which are a great disfigurement in a white dog. Here I may break off to remark that these marks wou'd also spoil white toy Poms, but for the fact that white toys of that breed are scarce. Breeders have done their best to get them, and a good many small ones - under 6 lbs. - have been bred, but the tiny whites shown are generally deficient in some point. Of toy 
whites, over $6 \mathrm{lbs}$. and under 8 lbs., there are now many, and good ; especially in a certain west-country kennel ; but some of the best are dangerously near the limit of weights.

The "tear-channels" which led to this digression can be helped not to exist by using a boracic acid lotion to the eye; but the stains are often ineffaceable.

\section{CHAPTER VIII}

\section{AILMENTS AND ILLNESSES}

Anæmia-a condition of general depression in health, with impoverishment of the blood-is of all serious diseases the most common among dogs. It is this condition that causes dogs to have worms; it is this deficiency in the blood supply, both in quantity and quality, which brings about ninety out of every hundred cases of skin disease. The original cause of the disease in toy dogs was the way in which they were, and unfortunately often still are, kept, fed, and housed. A number of dogs kept together in some artificially-heated building, confined in small pens, obliged to breathe impure air, and fed on Indian meal, biscuits, oatmeal, and other cereals, with little or no meat-this is kennel life, and a splendid foundation for anæmia. We all know how worms and eczema and other skin troubles beset toys kept " in kennels," but not until the knowledge has caused people to give up keeping them thus, and handing on hereditary eczema and hereditarily vitiated blood to their puppies, shall we get rid of the inherited tendency to poverty of blood which makes so many toy dogs possessions of anxiety rather than sources of satisfaction to their owners.

If a law could be passed obliging all dogs to receive a suitable daily allowance of good, fresh, underdone meat, and abolishing farinaceous feeding altogether, even for inve years, it is not too much to say that at the end of 
this time ezccma in its more common forms would have died out, worms be the infrequent exception rather than the rule, and "distcmper" would have ceased to be a thing of terror.

It is extraordinary how ignorant educated people, otherwise well informed, can show themselves on this subject. I have repeatedly received letters in which, after detailing a diet of milk puddings, oatmeal porridge, vegetables, bread and gravy, and so on, the writer gravely adds the assurance-" But I have never given a farinaceous diet!" Green vegetables and such starchy vegetables as potatoes are absolutely useless to dogs, and so indigestible as only to rank second to absolute poisons, like carrots and turnips. No dog can get the mineral salts necessary to healthy blood out of oatmeal, Indian corn meal, or any other meal, nor out of a little iron-hard, dried gristle or some similar substance, such as appears in some so-called "meat" foods. It can only get these substances out of its natural and proper food-meat. Puppies fed on meat from the time their teeth can bite it do not have anæmia, and are consequently free from skin trouble: their blood is rich and pure, and they do not harbour worms. I only ask any reader who doubts these statements to try the very simple experiment of separating a litter at seven weeks, and feeding half the pups on meat, of course varied, cut up small, and given in moderate quantity three times, and subsequently twice, a day, with a very small proportion of wheaten flour-stuff given merely as a treat and variety, in the form of small sweet biscuits or sponge cake, to afford the needful bulk to the meals. No gravy, milk, vegetables, nor any liquid but water to be given. The other pups in the litter can be fed on the old, artificial, unnatural plan of constant, large, sloppy meals of milk food. If the conditions are otherwise equal-plenty of fun, sunshine, and exercise being given-the difference between the two sets of pups will probably be quite sufficiently marked to uphold my argument, with the further addi- 
tion that the meat-fed puppies will be found a good deal less objectionable in the house before their education begins, and infinitely easier to train, than their brethren on farinaceous diet.

In cases of anæmia, as shown by skin trouble, bareness round the eyes, poor or capricious appetite, languor, unpleasant breath, thinness, and a general look of unthriftiness, a liberal meat diet is the first essential, and plenty of fresh air-not necessarily hard exercise, for which the patient is generally unfit-the next. A tonic is always desirable, and iron the most suitable. There are several forms of this useful drug. Reduced iron can be given in very small dosage; sulphate of iron is cheap and useful in pill form : both of these have a tendency to constipate. The saccharated carbonate of iron is a beautiful preparation that does not constipate-is, indeed, a little laxative in action. It is a powder, tasteless except for sweetness, and will be taken readily enough if sprinkled on meat, or it can be made into pills with the addition of a tonic bitter, as in the form of the Kanofelin tonic pills. It is the most expensive of the forms of iron, but that is not saying much, as all are absurdly low in price. The dose for a toy is from two to four grains twice a day, in, or immediately after, food. Cod liver oil is a useful medicine in bad cases of anæmia, especially where, by reason of having or having inherited, this habit of body, a longhaired toy is always poor in coat. Some dogs never grow coats, merely because they have not the strength to do so, and others inherit sparseness of hair. But if there is any hair in reserve, a course of cod liver oil will help it on, and better far than plain cod liver oil is its preparation with malt. Cheap cod liver oil, however, is horrid, and should never be given. It will only act as a purgative, and be worse than useless. Nor should a dog ever be forced to take this substance if he has a dislike to it. But if the anæmic, scantilycoated patient will take it readily, a teaspoonful of some good brand of cod liver oil and malt extract, besides 
three grains of saccharated carbonate of iron twice a day, with meat diet, will make a most marvellously different dog of him in six weeks' or two months' time.

It is quite useless to give any tonic for a week or ten days, or irregularly. It must be given for a long time and with perfect regularity, or it does no good whatever : it must have time to be absorbed into the system, to permeate it, and be taken up by the blood.

Bad Teeth.-The existence of canker in dogs' teeth is generally another consequence of bad rearing and farinaceous feeding. Meat - fed pups, from meat - fed. parents, have conspicuously good sound teeth, whereas among kennelled dogs it is not at all uncommon to find specimens of mouths cankered throughout, and this condition is certainly sometimes transmitted to the offspring. The teeth look deep yellow, or brown, the dental enamel is soft, and in bad cases they drop out. The gums are soft and spongy and pale. The disease being constitutional, little or nothing can be done to arrest the decay of the teeth, which luckily seems painless. The dog should be carefully fed on the most nutritious underdone meat, and the mouth may be washed out daily with a very weak solution of permanganate of potash: just enough of the crystals to tinge warm water pink being used. The best way to perform this little operation-one to which most dogs object very strongly-is to get someone to ho'd the head, with the nose pointing downwards, over a basin, and to introduce the nozzle of a gutta-percha ball syringe between the lips at the back of one side, letting it enter that spot in the jaw where there is a hiatus between the lower teeth. Two or three squeezes of the ball will then wash out the mouth pretty effectually.

This cankered condition of dogs' teeth may be brought about by the absorption of mercury into the system. A dcg which had been troubled with very obstinate recurrent eczema, known to be inherited from ill-reared parents, was apparently cured as by magic when sent 
to a veterinary surgeon, who dressed him all over with mercurial ointment. The improvement in his condition continued for about three months, when it was discovered that he ate with difficulty. His mouth being examined, the teeth, previously sound, were found to be like so much dark, yellow-brown leather, and the gums sore. The next development was in the form of a cancerous growth in the posterior nares, and so the poor animal died, a victim to a cruel " fake," for which the surgeon had obtained the credit of a cure. Such cases are not at all uncommon.

Dental Caries, such as affects our own teeth when they decay and have to be stopped, occasionally, though luckily not often, distresses dogs. They may bruise the dental pulp inside a tooth by biting very hard on a bone, or by playing too roughly, and more especially by carrying stones, a very bad practice. The only thing to be done is generally to extract the tooth under chloroform, since it is difficult to find dog-dentists who will stop a decayed tooth. A dog with toothache, rubbing his face on the ground and crying, is a pitiable sig!t.

Abscesses between or on the Toes are a form of eczema, and should be treated constitutionally, as suggested under the heading of Anæmia, eczema's usual cause. Dogs will worry these sores, and must be prevented from doing so by having the foot encased in a sock made of strong washed calico, tied round the leg with tape. Before putting on the sock, dress the sore with iodoform powder or zinc ointment.

Docking Puppies.-Being docked is not an ailment nor an illness, but as a very sad conclusion may be put to a valuable pup's life by the operation carelessly performed, it is as well to say a word about it. Docking should never be left until the eyes open and the nervous system is fully organized. At such an age it is a piece of gross cruelty and the risk of hæmorrhage is enormously increased. Unless puppies are very weakly, they 
should be docked at five days old at latest. Happy is the owner whose Poms or Pugs require no such improvement! The Schipperke owner has been especially commiserated or vituperated, as the case might be, but as a matter of fact there is, in the hands of a competent surgeon, used to operate on these and other dogs, not one iota more risk or more pain or more difficulty than in dealing with a terrier. Docking should be done by a skilled veterinary surgeon, with proper antiseptic precautions. His hands and the strong scissors used are first made thoroughly antiseptic by washing in carbolic or some other antiseptic solution, and the operation can be done without the pup's losing any blood at all to speak of. The wounds are dressed with iodoform powder and tannic acid powder, mixed, and in one hour the mother, who should be sent out for a walk while the surgeon is in the house, will be admitted to them, and they will be sucking as if nothing had happened. Occasionally, owing to some idiosyncrasy of the individual, a puppy may bleed after docking, and therefore a careful watch must always be kept. If there is any hæmorrhage, bathe with very cold water in which alum has been dissolved, and apply a styptic, as tannic acid or perchloride of iron. But it is always well to ask the operator to remain for an hour or so, until all risk is over. The bloodvessels very quickly seal up at their ends (to use untechnical language), and the tongue of the mother, when re-admitted after the necessary interval, will do no harm. Though docking is neither dangerous nor cruel when properly done on puppies so young that they have little or no sensation in their undeveloped nerves, it is a barbarism to let any ignorant person, as a groom or coachman, do it ; and the dog owner who will not sacrifice her own possible repugnance sufficiently to co-operate with the skilled surgeon in seeing it properly done, at least owes it as a duty to her dumb dependents to pay him to take all reasonable care, and bring an assistant to hold them, and stay until they are quite safe and comfortable. 
Bilious Attacks. - A slight chill, in east-windy times of year, or from any undue exposure to cold, will sometimes bring on a liver attack in dogs, while some are habitually subject to sick-headache after the manner of their owners. A bilious dog shivers, looks miserable, brings up a little yellow liquid or some froth, after a good deal of retching, and refuses to eat. Such an attack is always easy to diagnose, because the nose remains, as a rule, cold and moist, while there is no rise in temperature. The same symptoms, with feverishness, would probably mean commencing serious illness, necessitating skilled advice ; but without rise of temperature are not important, unless they resist treatment and continue for longer than about twelve hours. The patient should be kept warm, covered up before the fire if the weather is severe, and given a soft pill of three grains of carbonate of bismuth and one grain of bicarbonate of soda, every four hours, until appetite returns.

Loss of Appetite is a symptom which should never be disregarded. It may be quite right for the owners of sporting dogs to use the phrase so frequently heard : " Oh, if he won't eat, he's better without it," but want of appetite in a toy dog should never be a matter of indifference to the owner. It may, of course, arise only from previous over-eating, and over-fed dogs are certainly subject to bilious attacks which do not call for much sympathy; but it is always desirable to assure oneself that nothing more serious is the matter before dismissing the subject. In cases where loss of appetite is the precursor and accompaniment of illness, as in distemper, it would be most unwise to leave the dog to itself, and by allowing it to go without food, pull down the vitality and give the disease a firmer ho'd. As a general rule, a dog may be allowed to miss one meal without much anxiety; but, if a second is refused, inquisition should be made, and the temperature be taken, without loss of time. A clinical thermometer is a most useful adjunet in the dog-room, 
and any temperature over Ioo degs. or IOI degs. - the former the dog's normal one-is suspicious. The easiest way of taking it is by inserting the instrument between the thigh and the body, and, as it were, holding these together, over it. Puppies will often refuse food simply because their gums are sore from teething, and here, again, it would be extremely foolish to let them go on in a state of semi-starvation. When a puppy is seen to pick up his food with his front teeth, shake each piece, and turn it over indifferently, it is a pretty sure sign that he cannot eat comfortably; if the natural process of cutting the teeth is in fault, all that need be done is to give minced meat and soft though dry food-a sponge cake will nearly always be willingly negotiatedand keep a watch to see that he gets enough to maintain him in good condition and pull him through the critical time; if, as is sometimes the case with an older dog, a too-lingering first tooth is setting up irritation and needs extracting, the vet's services must be requisitioned, as it is not advisable for any amateur to try his hand at canine dentistry. The main characteristic of the " new" or Stuttgart disease, or of gastritis, by the way, is inability to take food, the mouth being ulcerated, in addition to stomach complications; and here, again, commencing loss of appetite must be regarded with suspicion. Simple biliousness is not common among properly-fed dogs, but is sometimes brought on in individuals by what I may be so technically medical as to call idiosyncrasy - to wit, inability to digest certain foods. Many toy dogs cannot eat vegetables, which of course are to all unnatural and very indigestible, and others are invariably sick if they are given milk, and the dog can no more help these peculiarities than human beings similarly afflicted. Biliousness, brought on either by over-eating, a chill on the liver, or some unsuitable food, is easily recognized, and here abstinence for a while is advisable. The patient will be chilly, probably having cold paws, and may be sick several times, producing only a little yellow 
froth; most dogs eat grass and soon feel better, r€ quiring no medicine; but if appetite does not return quickly, give a bismuth-and-soda pill every four hours, the proportion being three grains of bicarbonate of soda to one grain of carbonate of bismuth.

Indigestion is by no means uncommon among toy dogs, and frequently leads to the odious habit of eating horrible things in the street, about which dog owners sometimes complain, and with reason. The presence of worms leads up to this habit, too, and where it exists they may be first suspected; and then, if their existence is disproved, indigestion comes in as the likely factor. Its treatment is not difficult, but the owner must make up her mind to persevere, and to feed her dog herself-no servant, no matter how careful, possesses judgment enough to deal with a case of this kind. Absolute regularity in feeding is necessary; the meals must be small, yet very nourishing, and the dog should not be allowed to drink immediately after eating. A digestive tonic containing nux vomica is almost invariably useful, but it is not a medicine which can be prescribed at large, for nux vomica is in itself a dangerous drug, and acts much more freely upon some dogs than upon others, making it most unwise to prescribe "so much" for all dogs alike. With this proviso, I will give a prescription intended for a Yorkshire terrier weighing about 6 lbs., which may be safely tried upon toys between $5 \mathrm{lbs}$. and $8 \mathrm{lbs}$. weight, the quantity of this particular ingredient being reduced by one-half for dogs between 4 lbs. and $5 \mathrm{lbs}$. and by two-thirds for toy puppies, upon whom its administration must be watched with extra vigilance: $\mathbf{R}_{\boldsymbol{x}}$ pulv. nucis vom., $\frac{1}{2}$ gr.; pulv. radix gentianæ, I gr. ; carb. bismuthi, 4 grs. ; bicarb. sodii, $\mathrm{I} \frac{1}{2}$ grs. ; ferri carb. sar.ch., 3 grs. M. H. D. Exhib. cum cib. bis vel ter die. A pill somewhat similar, but in some respects superior to this, is sold as one of the Kanofelin remedies. 
The symptom of too great susceptibility to the action of strychnine (nux vomica) will be, in bold language, twitching and nervousness, and where these are observed to follow a dose it must be diminished or stopped altogether, and in this latter case the powder without the first ingredient may be tried.

Disagreeable Breath and Eructation.-Beta-naphthol, given in pills containing $\frac{1}{2} \mathrm{gr}$. each, is a valuable drug in cases of indigestion where eructation and disagreeable breath are noticeable. For toys under 5 lbs. $\frac{1}{4}$ gr. pills must be given; one pill in either case to be given about ten minutes after each meal. The effect of the drug is simply to check the fermentation of the food and the consequent formation of foul gases in the stomach. Where this form of indigestion is accompanied by diarrhœa, salol may be given instead of naphthol, in the same doses; but it and naphthol do not suit all dogs alike, though neither can do any harm, and if the patient is sick after a dose, the sign has been given that marks the treatment as unsuitable to his individuality. As in the case of human patients, the dog doctor may have to try several methods of treatment before he hits upon the cure. Pills are often troublesome to give, which fault cannot be found with powdered vegetable charcoal, to which few dogs make any objection when it is sprinkled upon their food and lightly covered with a few tiny bits of something very dainty; but where the owner prefers to give medicine apart from the food, enclosure of powder in a capsule is always practicable. A simple and tasteless powder is included among the Kanofelin Remedies, and may always have a trial, given with the food, in cases of indigestion.

The Bad Doer.-Want of appetite for no particular reason, except general debility of the stomach, is the annoying characteristic of the kennel-man's horror - the "bad doer," who is characterised by thinness and bad coat. Here and there we find a 
thin little $\operatorname{dog}^{-}$that nothing will fatten; hardly ever hungry, and dainty to the distraction of his owner; a dog who will not eat in a strange place or from an unusual plate, and who only grows the thinner and more miserable for what he does eat. $\mathrm{He}$ is an unenviable possession, but we must make the best of him, coax him with small and frequent meals, for he will often accept a teaspoonful of raw meat minced, or a tablespoonful of cream, where he would not even look at an ordinary dog's meal, and get him up as well as we can for show with a daily new-laid egg, beaten up in a very little milk, and that useful and valuable dogowner's aid, cod liver oil and malt. Most dogs will take this with a little tempting meat to help it down. Of course it must not be pushed at first, but given, to begin with, in very small doses, and gradually increased until our usefully typical $6 \mathrm{lb}$. dog is taking a full teaspoonful twice a day. It is a wonderful hair producer. Cod liver oil alone, without the malt, is of much less use, and cheap preparations of either or both are to be sternly avoided; in the nature of things, such a medicine cannot be cheap, if it is to be thoroughly good. And here, I may remark, that because we are only dealing with a dog is no reason why we should put cheap drugs of any kind into him. His system is just as beautiful and delicate in its balance as that of a human being, though his teeth and his digestion may be stronger-such is not invariably the case by any means-and the administration of impure or adulterated medicine is just as great a cruelty to it as to the human machinery. To give a toy dog crude cod liver oil, imperfectly purified, because it is cheap, is like expecting to do fine carving upon oak with a hatchet, because it is oak and not satin-wood.

Internal Parasites. - In no case has modern progress in knowledge disclosed more fallacies, held formerly as firm beliefs, than where the internal parasites-which for our present purpose, this being only a popular manual, 
may be classed as tape-worms and round worms-of the dog are concerned. Only a few years ago, if a dog suffered from skin disease in any one of its several forms, "worms" were at once cited as the cause. Now we know-or rather, those among us know, who either have some understanding of canine anatomy and physiology or will take the word of the scientist for it - that worms cause nothing: they are not a cause, but an effect. They are a symptom of anæmia; and as skin trouble almost invariably accompanies any severe degree of anæmia in dogs, skin trouble and worms are usually found together. We cannot, therefore, cure dogs of harbouring worms by giving expellent doses, no matter how glowingly advertised and boomed, of the various irritant drugs which act as vermifuges. We can only by this means temporarily drive out the enemy, which is certain to return, because the conditions prevailing in an anæmic intestine suit it perfectly, and encourage its increase, whereas in the healthy intestine it more or less shares the fate of food on being digested, and is incapable of rapid or sustained increase. The effect of an anæmic or vitiated condition of the blood-supply to the villi, or, in non-scientific language, digesting pores which exist all over the mucoid lining of the intestinal tract, is to prevent their throwing out those strong juices or digestive fluids which they normally produce. Their secretions are altered and weakened, and have no injurious effect on the parasites, which then increase rapidly. When, therefore, it becomes evident, by the appearance of short yellowish-white segments, generally about an inch long, and varying in breadth from a mere line to about a quarter of an inch, dropped about by a dog, that tape-worm exists ; or it is seen by his vomiting them up or otherwise, that he has round worms, which somewhat resemble earth-worms, what we have to do is to alter that condition of the general health which allows these pests to exist. In brief, we have to treat the dog for anæmia, which subject has been already discussed. It is, of course, occasionally pos- 
sible for a healthy, meat-fed dog to become accidentally infected by swallowing tape-worm ova, and in such a case a few of the parasites may be harboured for a considerable time, not increasing, but now and then making their presence manifest. Infection is possible by the swallowing of fleas, which are intermediate hosts of tape-worm, or by eating the insides of rabbits, which usually swarm with these creatures, or, in the opinion of some authorities, by sniffing the ova up through the nasal passages and subsequently swallowing them. As, however, one cannot always be certain that the apparently healthy dog is not a trifle below par, it is always well to treat him with a course of iron, giving the powders or tonic pills advised for anæmia for a month, and at the expiration of that period, when the system is toned up so that the worms' position is almost untenable, and their expulsion will be final, one or two vermifuge doses may be given. All sorts of quack remedies have been praised and boomed as infallible, but many are exceedingly drastic, and some positively dangerous. Areca nut, so frequently advised, is a most violent irritant, actually poisonous in its effects on young puppies, and a very cruel remedy in all cases. Wormseed oil, an American preparation, possibly from one of the inulas, a family of plants known in English gardens, is sometimes an ingredient; also such highly unsuitable, inert, useless, or dangerous substances as sulphate of magnesia, salt, or cowhage, with strong doses of santonine, a drug that should never be given in unknown quantity. A violent purgative action often accompanies these secret remedies, adding to their danger. The intelligent dog owner should know what he is giving, and to some extent understand its action; but in a country where quack, much-advertised medicines are largely given to children, I suppose it will be difficult to prevent their being also administered to dogs. In any case, no worm medicine whatever, of any sort or kind, other than an iron tonic, should be given to young puppies, no known drug possessing a stronger action 
than iron upon the parasites being safe for toy pups under three months old. After that age it is safe to give very small doses of oil of male-fern and absolutely minute ones of santonine. These are best combined in a capsule, in which form they can be given without distressing the patient, and a perfectly safe capsule after this formula is, among the Kanofelin remedies-which are not secret, but are compounded after recognised formulæ, and equally suitable for dogs or children in the purity of their drugs and safety of their action. If any of the popular advertised remedies are used for adults, experiment should be made at first with much smaller doses than are cited, and safety thus assured, for a microscopic dose will often act quite severely enough for the toy dog owner's purpose, and dogs are as variously sensitive to drug action as we ourselves.

In very young puppies the bringing up by the mouth of round worms is not at all unusual, especially when they are pups born of " kennel " parents, dogs crowded together in numbers, insufficiently fed (although possibly upon an excessive quantity of oatmeal and Indian corn meal), denied meat, and leading a completely unnatural life in every respect. It is rather a shock to an amateur when this occurs, but as a rule little anxiety need be felt, for if the puppy is properly fed upon small dry meals of a very digestible and nourishing nature, say two tablespoonfuls of good underdone rump-steak, or the same quantity of roast mutton, three times a day for a dog the size of a pug, and given a one-grain dose of iron with two of these meals, he will be pretty sure to grow out of his troubles. In any such case great attention must be paid to keeping up the strength of the patient, in order to tide him over the time when by reason of youth and his very tender little stomach, it is impossible to give him any stronger medicine with safety.

Extreme thinness and loss of coat are sometimes attributed to that wonderful power worms, in oldfashioned eyes, possessed. Both of these symptoms 
are those of an anæmic condition, as is fœtor of the breath. Finally, the treatment of that over-rated bugbear in the way of diseases, "Worms," is easily summarised thus - Meat feeding; an iron tonic; a vermifuge after the tonic course, and not before.

After male-fern capsules it is quite unnecessary to give any aperient. Most inventors of "worm pills" and the like order castor oil to be given after their boluses, a terrible aggravation both to operator and patient.

Aperients. - Some people have an idea that it is desirable to dose dogs periodically, on the quaint old " springmedicine" principle, extended over all the year. No greater mistake can be made. A dog should never be given drugs of any kind unless really ill, and this it will never be in the direction indicated, if it is properly fed and regularly exercised. A dog's natural and proper food is meat; but the stimulus of distension must be given to the intestine by adding some bulk of innutritious food to the meat. We cannot give quite enough meat to afford this stimulus constantly, because by doing so we should overload the system. In a state of nature dogs ate the fur and skins of their prey, like other carnivora : now we must give them a certain proportion, but only a small one, of biscuits made of wheat not of oatmeal or Indian corn meal, which are too indigestible) or of brown bread, to provide bulk without nourishment. They may, if any aperient be absolutely necessary, have a meal of boiled liver, a teaspoonful or two of pure olive oil poured over a little meat, or given from a spoon, or some cod liver oil, which máy be voluntarily taken, and is equally efficacious. Milk is very laxative, and sometimes, where there is no biliousness, a small saucerful makes a good apcrient. Always take a dog for his run at the same time of day, wet or fine, and never lose sight of the fact that a wellbehaved clean little house-pet may bring upon itself a dangerous attack of constipation by its good manners if its appeal for a walk is ignored. 
Distemper.-As a matter of actual fact, there is no such disease as distemper. There are two diseases, or two groups of diseases, both more or less contagious, which, for want of skilled diagnosis, are indifferently so named, but their popular designation is so firmly rooted that " distemper" will be with us to the end of the chapter, and so long as the disease is properly treated it matters little whether we call it bronchial catarrh, gastro-enteritis, typhoid, or distemper. Per-

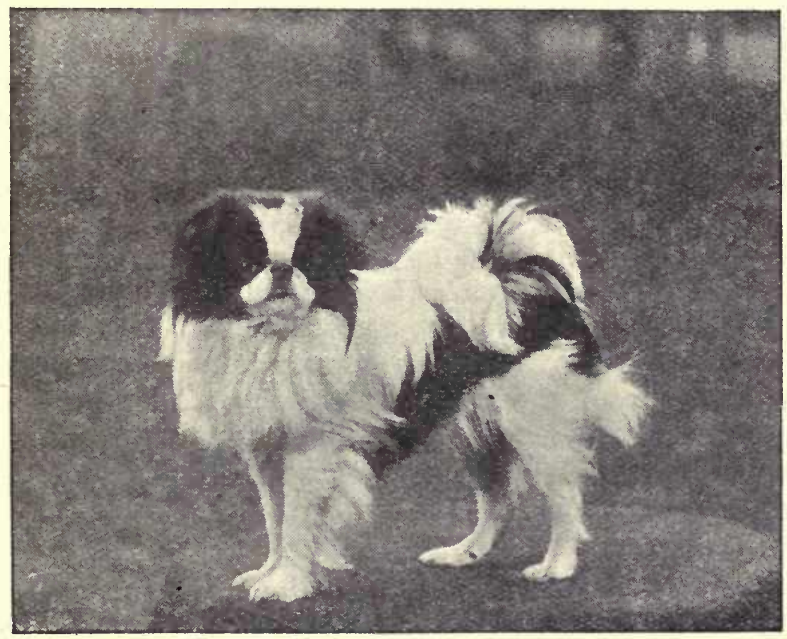

TYPICAL JAPANESE SPANIEL.

haps, in a manual not intended for the learned, it will be most useful, as it is certainly most simple, and, I think, practical, to speak of "two forms of distemper," since the chest and lung diseases of the dog all call for one sort of home treatment, and the more ordinary diseases of the intestinal tract can with safety be lumped together as needing another fairly uniform style of treatment. Further than this the non-medical dog owner is not wise to venture, since it is quite as necessary 
that a canine patient should have skilled advice as that it should be called in for his master-that is, if his recovery is desired.

Roughly speaking, then, there are two kinds of distemper-that which affects the nose, throat, and chest, and in slight cases may pass as being only a very bad co'd, and that which affects the intestinal canal, involving the whole alimentary system. This latter is certainly the more troublesome for an amateur to treat, and decidedly the more fatal; but, fortunately, the former is the more common. It is very easy to tell when a dog is the subject of distemper in the catarrhal form, and when in this state he is, I think, much more likely to do well if carefully nursed at home ; but in the typhoid form it requires skilled nursing to do the case justice, and the physical conditions are such that if-it is a big." if "- the right sort of vet can be found, the dog has a better chance with him.

The symptoms of catarrhal distemper are shivering, feverishness-temperature generally not very high at first, but a degree or two over the normal-profuse discharge from the eyes and nose, and, in short, all those of a bad, feverish cold ; and the treatment may be exactly that which we should give a child under the same circumstances. The great thing, in both forms, is to keep up the strength from the very beginning; this is far more important than giving medicine of any kind, and if the patient will not eat, he should be given food forcibly. I do not by this mean that a large quantity of food should be forced upon the unwilling animal ; he should have about two teaspoonfuls of some invalid nourishment every two hours, and this should be as varied as possible, and kept as sweet and dainty as if for a human patient. A raw egg beaten up with the smallest possible quantity of milk; a little good beef-tea, made by cutting lean, raw beef into small cubes, and slowly drawing all the goodness out of it in an earthenware jar, tightly covered, in the oven, only two tablespoonfuls of water to the pound of 
meat being added; veal broth similarly made; arrowroot, with a few drops of the juice of raw meat added ; strong chicken tea, with a little rice boiled in it and strained out-all these may be rung upon for change. Some dogs will eat solid food all through the disease, and this simplifies matters immensely. Where there is no appetite, liquids or semi-liquids must be given. Concentrated foods and other invalid preparations, though useful on occasion, very soon pall and sicken the patient, and while it saves trouble to use things like this, they have not the same effect in keeping up the strength as good, honest home-cookery. The necessity for thus dieting and feeding is the same in either form of distemper, and the dog must not be left all night without attention, but fed at intervals then also. Warmth and evenness of temperature come next in importance. A little flannel jacket or crossover, made of thick, new flannel, is as good as poultices, and should be put, and kept, on well into convalescence, when, of course, it must not be left off too suddenly. I do not say anything about medicine, actual poulticing, etc., because a distemper patient, in view of the complications which are always apt to arise in this disease, should be nursed under skilled veterinary direction. I only insist on the need for feeding up and warmth.

Distemper patients cannot go out of doors, in cold weather, unless there is to be no regard to the great risk they run in such a change of temperature; therefore, as soon as the disease declares itself, it is well to settle the patient somewhere where a tray of earth can be provided, absolute quiet maintained, and an even warmth kept up, and here let the disease run its course.

Relapses from distemper are even more serious than the first attack, and they are very apt to occur where the patient is allowed to go out, or move about too soon or too much. Stimulants - brandy and port wine -are very useful where the weakness is great, and champagne will often be kept down where water or broth would be rejected. 
The " new" disease, commonly called the Stuttgart disease, which has created so much excitement among dog owners during the last year or two, and is of the nature of gastritis, or inflammation of the lining membrane of the stomach, spreading upwards and downwards, calls in some ways for quite a different treatment to that of the typhoid form of distemper. They are alike in this : that a teaspoonful or so of iced champagne or iced soda and milk, will sometimes be retained where nothing else will, but in gastric catarrh, or gastritis, the patient must not be allowed to drink water, or to make the slightest exertion.

It may, perhaps, be as well to state what, I suppose, is not yet known to all dog owners-namely, the fact that it is by no means a necessity for a toy, or any other dog for that matter, to have distemper. Like scarlet fever in the human subject, distemper may occur in a dog's life, or may not. The child takes scarlet fever if it has been in the way of infection, and the dog distemper if the contagion has been conveyed to it either by some person who has been near an affected dog, by that dog itself, or by some article on which infected discharges of any kind have been deposited.

The one quarrel we all have with shows is that they certainly offer opportunities of spreading distemper to people who do not consider its existence in their kennels a sufficient reason for withholding entries, and carry the contagion with them, although the dogs they exhibit may be in themselves unaffected. An old-fashioned piece of advice in distemper, and one always given, was that at the outset of the disease a dose of castor oil, or some other aperient, should be administered. I have no hesitation at all in saying that whereas castor oil - to the dog a violent irritant purgative-has carried off many and many a puppy and delicate adult that, if not so weakened just when all the reserve forces of strength were most needed, might have pulled through, this practice is a most mistaken one, to say the least of it. If there is any probability of there being any collection in the 
intestine which needs clearing away, pure olive oil will do all, and more than castor oil, and will neither cause the pain at the time nor the subsequent constipation, which will be the inevitable results, if there are no worse ones, of the stronger, and, I must call it, vile, drug. Another fallacy is the supposed desirability of constantly washing the eyes and nose with warm water. This is often not properly dried off, and chill results, while all the fuss and worry is quite needless and does no good. A little bit of old linen rag may be torn up and the fragments used to clean off the discharges and at once burnt. Once, or even twice, a day a sponge damped with boracic lotion can be used, but very sparingly.

The watchword in distemper, as I said before, is nursing-good nursing alone will pull most dogs through - and I deliberately refrain from giving any prescriptions, because, as each case varies according to circumstances and the patient's constitution, each should be prescribed for on its merits.

For far too long we have gone on in a rough-andready rule-of-thumb method of dosing dogs all in the same way, without regard to idiosyncrasy, which all the time has been as marked in them as in human kind-and the sooner we change all this and study each dog after its kind, the better for them and for us.

Skin Troubles.-The most annoying thing about the skin complaints which occasionally beset toy dogs is the difficulty to the amateur of diagnosing them correctly. Even veterinary surgeons are sometimes hazy in this respect, and it is therefore well when a skin trouble refuses to yield to simple remedies, incapable of doing harm, to consult a man really experienced in toys, and not some uninterested, and even rather contemptuous, practitioner, who may even commit such a cruel barbarity as I have heard of, in the advising of sheep dip!

The most common form of skin disease in adult 
dogs is eczema, which for purposes of rough, or popular, classification, may be divided into two forms, wet and dry. Weeping eczema is decidedly uncommon, but is the only form of skin disease offering open sores and raw surfaces likely to affect comparatively well-caredfor toy dogs. In this, as in the dry, severer forms of eczema, it is useless to attempt cure by mere outward applications. The mischief is in the blood, and until the blood is put right the external symptoms will continue, unless, indeed, strong mercurial lotion or ointment be used, which may fatally drive the disease in, and by clearing up the skin and so depriving the body of the safety-valve of outward lesions, eventually kill the animal. Such a proceeding is occasionally resorted to by unscrupulous persons whose only desire is to sell their mangy or eczematous dogs, for the immediate effect of dressing with mercurial ointment is often almost miraculously good to the eye. Therefore, my advice to the amateur is, under no circumstances to purchase a dog which is known to have suffered from any severe form of skin disease. Even if the complaint has not been doctored in the way described, and has been cured by honest methods, it may always break out again, for it is in the constitution. I must, of course, except cases in which contagious eczema has been given to the victim by some other dog, but in dealing with strangers, shops, or professional dealers, it is wisest to avoid a purchase where skin disease has existed.

Some breeds are very much more subject to skin trouble than others, and all long-haired dogs are apt to suffer from simple eczema and erythema, the latter especially when young; while distemper of a severe kind is often followed by a disease of the skin, closely resembling mange, for which it is often unfortunately mistaken. It should be simply treated with a mild antiseptic ointment, while the constitutional weakness is the focus for attention.

Puppies often teeth with a rash, called puppy-pox, 
which shows as general redness of the skin, generally on the bare parts of the body, under the forelegs, etc., and here and there groups of pustules, each of which contains a drop of thin pus. This is a complaint allied to chicken-pox in children, and by no means dangerous -in fact, a puppy which teethes with such a rash has generally the making of a strong and healthy dog. At the same time, whenever either this trouble, or bare patches about the legs and face, are seen on puppies, the teeth should be looked to, for it is probable they are in some way irritating the system.

The existence of too many worms in puppies generally accompanies skin trouble in the form of bare patches, which may be well rubbed daily with a sponge dipped in an extremely simple, safe, and useful lotion, which I can recommend to be given a trial in all forms of skin disease, as in no case can it do harm, while in many cases it will effect a cure so far as any outward application is capable of doing. It is known as the Kanofelin lotion, a preparation of phenyl, which is not irritating, or in any way poisonous or disagreeable to the nose, but has a taste which prevents dogs from licking it off ; should they do so, however, it will not harm them. The lotion, after being applied and well rubbed in with the sponge to smooth, bare places, where the skin is not broken, should be wiped off with a towel or handkerchief, as it is not wise to leave the dog wet. It should be used twice a day, and where the skin is broken, very gently with a soft sponge, and, of course, no rubbing in.

Some dry and scaly skin eruptions, of which pityriasis is the most common, need different treatment. Whereever bare places appearing on the toy dog look scurfy, and scales fall off, do not use any lotion, nor rub, but lightly dab on a little zinc ointment if the dog is not given to licking the parts ; if he is, use a plain, rather thin, sulphur ointment: Sublimated sulphur, I oz.; vaseline, 4 ozs. This latter may also be used in cases where the Kanofelin lotion is useful, and then be well 
rubbed in ; but the rule is no rubbing when scales or scurf are present. The Kanofelin ointment is harmless and useful in all cases. Applications can be much varied to suit cases, and where violent irritation is present, it is sometimes necessary to use a more complex preparation than those mentioned. The poisonous nature of some of the ingredients, included in the most efficacious of them, however, makes it very undesirable to use them otherwise than under the advice of a skilled surgeon. The following cream is a most useful application for use in cases where the skin is not broken, where great irritation and redness of the skin are present, and where the affected parts either cannot be reached by the patient, or the latter can be muzzled during treatment. It is, however, poisonous, on account of the carbolic acid and lead it contains: Liquor plumbi diacet., 4 drs.; liquor carbonis detergens, $40 \mathrm{mns}$; boracic acid powder, I oz. ; new milk, to 4 ozs. Shake well before use, and apply frequently with a bit of sponge. Label : Poison.

In the treatment of medicated baths, usually composed of that most evil-smelling compound liver of sulphur and water - in professional language, " a sulphuretted potash solution "-I own I have little or no faith. A plain sulphur ointment is twice as efficacious, far easier to apply, and has no disagreeable smell; while, if well rubbed into the skin, as it and other skin ointments should be, and not left in the hair, it is not in any way unpleasant.

In all cases where skin trouble is accompanied by a strong and most unpleasant smell, mange (either follicular, or, more commonly, sarcoptic), may be suspected. The latter is easier to cure than many forms of eczema, but it is absolutely needful to keep the patient smothered in a dressing of sweet oil and sulphur, than which there is nothing better, for several days, then to wash and dress again; and such cases are not suitable for home treatment, although no veterinary surgeon should be permitted to apply strong dressings like 
paraffin, mercurial ointment, or tar (otherwise creosote) to delicate toys. Mercurial dressings, in all cases, are rank poison, the absorption of the drug into the system having fatal effects for the future.

Follicular mange, in which the insect causing the trouble burrows deep, is a horrible disease, about the worst a dog can have, and here skilled veterinary assistance cannot be dispensed with. But it is safe for the amateur, in all cases of commencing skin trouble, where there is no smell and the bare patches do not spread rapidly, to use the phenyl lotion or sulphur or Kanofelin ointment, according to the state of the skin, and to begin the more important internal treatment by a complete change of diet.

A very dry or confined diet, certain meals, as oatmeal or Indian corn meals, either in biscuits or otherwise; too little food ; more rarely too much; absence of meat from the dietary, or too little of it ; as before, but very rarely too much - these are all incentives to skin trouble, while heredity has much to say to a tendency thereto.

A dog which has not been having much meat, but has been chiefly fed on dog biscuit, may, on the appearance of skin irritation, be given plenty of good, underdone meat-roast mutton, sheep's head, and bullock's heart, all being very suitable. In no case of skin disease should either oatmeal or Indian corn be given; and sea air should be avoided, as it is always aggravating to skin troubles. Tripe is nourishing and very digestible, and fresh fish suits most of the invalids very well. Together with the entire change of diet-the hours for meals need not, of course, be altered-a course of iron and cod liver oil is always well worth trying. Personally, I pin my faith to the following method, which I have known most successful in difficult cases, and which, as I can say of the other remedies advised in this little book, can do no harm. Powerful drugs are often a source of danger in inexperienced hands, and a good many of the medicines one sees advised are, so to speak, extremely speculative. 
Get, then, a bottle of cod liver oil and malt, and I oz.-or more, if you please-of saccharated carbonate of iron. In your pet's dinner mix, at first, well covered over with cut-up meat of extra daintiness, a scant half-teaspoonful of the solution with a dust of the iron, which is a sweet powder. Nearly all dogs will take this without any trouble, and soon get very fond of the oil, even if they object to it at first; but they must not see the dose introduced into the meal. Let them think it an accident, or at any rate, in the natural way of things, and they are far less likely to object than if they see you making a parade of mixing and covering. The dose, given twice a day, in meat dinner and supper, should be gradually increased, until a dog of $6 \mathrm{lbs}$. is taking a full teaspoonful of the solution twice a day, with 3 grs. of iron to each dose ; and patience will be needed, for, to do any good, this dosing must go on for at least a month. It may then be left off gradually, and resumed again if necessary. In obstinate cases of skin disease, arsenic is a most valuable remedy, and may with most effect be combined with the system of cod liver oil, malt extract, and saccharated carbonate of iron just described. Fowler's solution, which is generally recommended, should not be used, because it contains oil of lavender, which is very offensive to dogs, and sickens them; the British Pharmacopœia solution should be the one used. Of this the dose is from one drop twice a day, to be gradually increased up to four drops twice a day for toys; the best way is to get the B.P. solution from your chemist, mixed with such a quantity of distilled water as that there are four drops in each teaspoonful. This may be given with iron and without the cod liver oil, or with cod liver oil without the iron, or alone, in food-it is tasteless-but is far better given in combination with the two. Mr. Appleby, Argyle Street, Bath, puts up the iron and arsenic together in a very easily used form, known as the "Kanofelin Blood Mixture," This, my own formula, I generally advise 
to my readers whose dogs do not or cannot take cod liver oil ; he also, inter alia, puts up the worm capsules to my prescription as mentioned for the use of toy dog owners ; and it is sometimes an advantage to get your medicines ready made.

Arsenic is what is known as a cumulative drug; it produces no special effect until a good deal is stored up in the system. When enough has been given, the said system revolts, and now, when the dog's eyes begin to look watery, and the mucous membrane lining the mouth may be a little red, you have given enough, and must cease ; for a time only if the disease is not subdued -in permanence if it be. One last word--arsenic is the dernier ressort, and should not be used until other means have failed, whereas some people fly to it when a much simpler treatment would have done all that was necessary.

Another skin complaint which is much more common than is generally supposed, is ringworm. I have often seen this diagnosed as eczema, whereas it really is very easy to tell its true nature, as it has very marked characteristics.

It begins with tiny, round, bare spots, about as large as the head of a pin, which usually escape notice at first, but gradually spread round the edges, not always in a circular form, but sometimes as irregular patches, the skin appearing greyish, but not unhealthy. On looking closely it will be seen that the hairs have been broken off short, close to the skin, but are clearly visible, which is the chief feature of the disease and the infallible sign. Ringworm may be caught at any time, most frequently from a visit to some infested stable, but occasionally from chance contagion in the streets. Horses are subject to the same form of the complaint, and dogs generally catch it from them; it is sporadic, and the spores may, of course, fall about anywhere from an infected horse or another dog. It is extremely capricious in its inception; dogs in the same house may or may not catch it from one another, and sometimes a whole kennel will be infected, with the exception 
- of one or two dogs apparently immune. There is, however, no excuse for allowing it to spread, as it is easy to cure. Some of the strongest tincture of iodine available should be well soaked into the spot, and round the edges thereof, using a little ball of cotton wool tied on to the end of a tiny stick, or an aural sponge, and rubbing the iodine somewhat in with this. Two applications will generally kill the spores-the disease is a parasitic fungus - and should be made at an interval of a couple of days. For some time fresh spots are likely to appear, and should be touched up at once. The muzzle, legs, and chest are generally most affected. If left quite alone the complaint would disfigure the dog terribly, but would, after a time, die out of its own accord. I have not found that human subjects were infected with this disease from the dog. A little iodide of potassium ointment may be put on the patches once or twice, to hasten the complete cure, or they may be washed with the phenyl lotion, in which the proportion is I in 40. The hairs are weakened, and take some little time to grow properly again, but the disease is by no means a serious one, and it is not necessary to use any such stronger and dangerous remedies as carbolic acid, as sometimes suggested.

Erythema, a general redness and rash, most often seen over the inside of the thighs, and sometimes all over a dog's least hairy parts, is about the only skin disease -if we except the curious and rare condition, "hidebound"-from which dogs very occasionally suffer, that, in a common way, arises from over-feeding. It is best treated by change of diet, small nourishing meat meals, and the avoidance of any heating, farinaceous substances, milk, or greasy food of any kind. A small dose of sulphate of magnesia twice a week in food-as much as will lie, not heaped, on sixpence for a 6-1b. dogis often all the medicine needful. Want of exercise is a frequent producer of skin disease. Dogs not sufficiently exercised, or kept much shut up in hot rooms, have inactive livers, whence all kinds of evils. 
I have never seen but one case of "hide-bound " in a house-dog, and that not in a toy. The skin was thickened and hard. Although the complaint is an interesting one from its rarity, that same fortunate quality renders it unnecessary for me to enter into the questiona veterinary surgeon must undertake such a case.

The Ears.- The ears in toy dogs are often the seat of a slight congestion which has no particular cause, but is more common in some individuals than others, and generally occurs at intervals in those subjects which have once had it. If taken early, the cure of an attack is very simple ; but if neglected, the congested state may increase and culminate in inflammation of the middle ear, otitis, and the bugbear " canker," of which we hear so much, and which is really extremely rare. There are many stages of the trouble, from the slightly hot and red external ear, which causes the dog to put two claws in the passage and try to scratch it, and sometimes succeed in making a sore place thereby, through the phases of rubbing the side of the head on the carpet or ground, groaning and shaking the head violently, and other manifestations of pain, up to the existence of real canker, when there is much soreness and redness externally, with swelling of the meatus, or passage, a profuse and very dark brown discharge, and a very disagreeable odour.

There is always a slight characteristic smell about a " bad ear," which any experienced person can recognise in an instant, often before any other sign of trouble is seen. Some dogs-most, in fact-need watching in this respect. The moment the toy is seen to be a little one-sided as to head, or evinces any disposition to scratch his ear, a small lump of boric ointment should be put in the meatus, pushed in with the little finger, and worked about until it melts down into the passage and convolutions. Next day the ear may be cleaned out with the tip of the little finger covered with a very soft handkerchief, and the ointment again used, and this, in slight 
cases, will effect a cure. Never attempt to put any hard instrument, or, indeed, any instrument at all, other than the soft suppleness of a feeling finger, into a dog's ear.

If the trouble has gone on a good while, and there is much brown discharge, it will be necessary to use a lotion. First of all use the ointment, as described, and clear away as much of the softened discharge as possible by this means, being, of course, exceedingly gentle in your manipulation, for these, at best, are very tender parts. Then take the following lotion: Warm water, $\frac{1}{2}$ pt. ; Goulard's extract of lead, I tablespoonful ; powdored boracic acid, $\frac{1}{2} \mathrm{dr}$. The boracic powder to be added to the water first, and the Goulard after, and the whole on no account to be used otherwise than nicely warm, or it will cause pain. The bottle can, of course, be filled at once, and a little of the contents warmed for use as needed. Lay the patient down on the sound side, with the bad ear uppermost, and get someone to hold him firmly. Then gently pour about half to one teaspoonful of the warm lotion into the ear, and work it about from outside. Keep him lying still for three or five minutes, then let him go, and fly! For he will shake the superfluous lotion all over you if you are not cautious. A great deal of remonstrant ploughing about generally follows, but the application does not really cause any pain, and will soon cure if persevered with-twice a day for a week or so. Such frightful and almost, if not quite, incurable cases as one sometimes meets with in sporting dogs, where the ears have become thoroughly diseased from, in the first place, getting wet and dirty, and being subsequently neglected, are, I rejoice to say, unknown among well-cared-for toys.

People are sometimes alarmed because their puppies' ears do not stand erect when they should, or are pointing ing all directions but the right when they should drop. This is a common thing enough during teething, and will generally come quite right later on. If it does not, no active remedy-by operation-is permissible 
if the dog is to be shown, but a good deal can be done by oiling the ears and manipulating them constantly in the desired direction by massage, while, in the case of youngish puppies, two or three thicknesses of horses' leg bandage plaster, cut to fit the inside and point of the ear, will either, if stuck in by warming it, help the ear to drop or to stand up, as is desired. This is a legitimate "fake," I may remark. But, of course, the process must not be used with any idea of deception, though it is allowable to aid Nature in the way she should go.

The Eyes.-The eye of the dog is an even more delicate structure than the ear, and only skilled surgical aid should approach it in any but the simplest ailments. Of these are the simple catarrhal ophthalmia, the symptoms of which are redness of the lining membrane of the lids, and a greenish discharge, turning brown and dry later, which comes from cold and weakness of constitution. The victim of this must be kept in an even temperature, be not allowed to lie by the fire, or look into it, or to go out of doors in wind, hot sunshine, or cold, and be well fed with good nourishing meat and light, digestible food. The discharge should be wiped away from the eyes at morning and evening with a bit of sponge dipped in a warm boracic lotion which any chemist will supply of the proper strength; and immediately afterwards a little bit of yellow oxide of mercury ointment, about as large as a small split pea, should be gently introduced under the lid of the affected eye with a camel's hair brush. Do not, on any account, accept " golden ointment," if the chemist happens to offer you this old-fashioned remedy (I believe) for styes ! It is made of the red oxide of mercury, and is a very great deal stronger than the yellow oxide of mercury ointment, which, by the way, should be made in the strength of 2 grs. to the ounce. This latter ointment may also be used where, after distemper, a bluish film lingers in the eye. Amaurosis is not 
uncommon in the dog. The eyes look perfectly right, but the dog is blind. This may be an hereditary condition, but sometimes comes in as a result of weakness pure and simple. Iron tonics, cod liver oil, nux vomica, etc, may be given, and sometimes prove effectual. Good living is essential. These cases are occasionally cured rather suddenly, but as a rule are incurable.

Simple cold in the eyes-or more often, only in one -is a very ordinary ailment, but distressing both to sufferer and owner. The affected eye waters more or less profusely, and is kept partly closed. Within, there is the same appearance as in catarrhal ophthalmia, but in a less degree, and there may be fever and constitutional disturbance, in which case the patient must be treated for a coryza, or " common cold." A boracic and poppy-head lotion is the quickest cure for cold in the eyes, and is also useful in the ophthalmic condition. It soothes the pain greatly, and is best applied by means of a small all-indiarubber ball syringe. On no account must a syringe with a bone or glass or vulcanite point be used : the indiarubber nozzle is soft, and from it one or two drops can easily be inserted between the eyelids. The amount of resistance the patient makes will be proportionate to the severity of the inflammation, and as this lessens he will endure the operation with serenity. To make the lotion at home, buy a poppy-head, price about a halfpenny, from any chemist, and boil it for an hour or longer in half a pint of water, adding to this as it evaporates. When the water is sherry-coloured, dissolve Io grs. of boracic acid powder in each fluid ounce, allow to cool, and use as frequently as convenient-once every hour, while the congestion of the lining membrane of the eyelids is active.

Sore Feet.-Eczema, or little boils between the toes and round the dew-claw on the front legs, is a trouble which besets some dogs. Constitutional treat- 
ment, as laid down for eczema, is needful, and as the dog will invariably worry the sores incessantly by licking, they should be dusted with zinc or ichthyol powder, and then bandaged or socked. If a dog is constantly licking its dew-claw, look at it to make sure it is not growing in. In this case it needs to be cut rather short, preferably by a veterinary surgeon, and the sore dressed. Dew-claws on the hind legs should always be removed by a veterinary surgeon in puppyhood.

Colds and Coughs.-Colds, or coryza, beset dogs as they do humans, but in lesser degree. A chest cold needs a flannel cross-over, sometimes a hot linseed poultice (in treating dogs it is much better to use, if possible, some dry poultice which will not leave the dog sopping after it is removed), or a mustard-leat. Rubbing with white vaseline oil and ten drops of turpentine to each ounce, if vigorously done, is as good for colds as for rheumatism. Everyone knows what a cold is, and the toy dog's cold should be treated like one's own. The clinical thermometer should be used, and if the temperature exceeds $100^{\circ}$, a pill of $5 \mathrm{grs}$. of nitrate of potash should be given every four hours until it is normal again, or, if it cannot be got down thus, give $\frac{1}{2} \mathrm{gr}$. of sulphate of quinine and I gr. of phenacetin, using the tabloids, and dividing them as desired. The strength must be well kept up. Coughsthe dog's hollow, deep-drawn brand-are a sore trial to the hearer. They sound terrible, but are seldom of much moment. If from cold, put a little vaseline or giycerine on the nose three or four times a day. It will be licked off, and give relief, while some dogs will eat glycerine lozenges if not flavoured with lemon. Vaseline, again, is an excellent thing for bronchial wheezing, such as pugs are especially subject to, and will always be taken if put on the nose. Cream also is soothing, and where is the dog that does not like it ? 
Chest Diseases.-The worst-sounding coughs are often the least important, and may pass off in a few days without treatment, but a bronchial rattling in the throat calls for care. Bronchitis in toy dogs must be treated exactly as in children, and, needless to say, the dog must not go out until the acute stage is passed. Most clean dogs will go to a box of earth in a cellar. A bronchitis kettle must be kept going in the room, and the patient will need an invalidish diet and much petting and amusement to carry him through the dull hours of discomfort. Dogs have congestion of the lungs, pleurisy, pneumonia, just as people do, and need the same careful nursing. Medicine in such cases is usually unnecessary, because it worries the patient and can do little good. A mild fever mixture may be prescribed by the vet, who should always be called in the moment the breathing goes wrong. Dulness, lassitude, shivering, and a high temperature-the clinical thermometer is of all things needed here-with troubled breathing, are symptoms of the highest importance, and skilled aid should be immediately called to them, The amateur cannot diagnose these lung and chest troubles.

Stomach Coughs.-Very dreadful coughs are sometimes heard proceeding entirely from the stomach. For these a little course of indigestion treatment often does wonders. Or, again, coughing may be caused by a fish-bone or something similar in the throat, though this is the rarest of all causes in the dog, owing to his possessing a most tremendous gullet, quite out of proportion to his size.

Shivering.--Shivering is a bad trick some dogs acquire, and others have by nature. It generally, if unaccompanied by a high temperature, means nothing whatever, unless it be nerves. But, short of the Weir Mitchell treatment, I imagine nothing benefits these latter more than a mild scolding, with admonitions " not to be so silly." 
Hysteria.-There are, most certainly, hysterical dogs, and their temperament is that of the habitual shiverer, though very thin-skinned toys sometimes really shiver from cold. A hysterical dog will bark itself quite out of breath at the least disturbance, and shriek exactly like its prototype human. Nature cannot be changed, but a tonic sometimes does good. Excitability and nervousness are characteristic of some breeds. Poms are, perhaps, the most excitable of small dogs, and pugs certainly the least so.

Obesity.-Extreme fatness may be a disease in the dog as in the human being, and in this case it is cruel to accuse the poor creature of systematic over-eating, as it is everyone's impulse to do. The bromides and iodides are useful, but cannot be prescribed haphazard. Thyroid gland tabloids may also be tried, beginning with one once a day, and gradually creeping up to three a day, according to the dog's size. Their effect on the digestion is not always happy, so that the dog must be watched to assure the owner of its toleration of them.

Poison.- -Not an ailment, but a subject which needs a few words, is the taking of poison by toy dogs. Unluckily, there is always risk in a town, not only of the wilful poisoner, who apparently exists, but of the ingestion of poisoned meat or bread and butter put for rats or beetles, and afterwards thrown out. In ninetynine cases out of a hundred a poisoned dog has had strychnine, this being the favourite drug of all those who employ poison at all. Arsenic is too slow, and of other poisons, thank Providence! the vulgar have mostly no knowledge. The symptoms of strychnine poisoning are, firstly, excitement-the patient runs about, and barks with a peculiar strident shriek. According to the quantity of the poison taken and the quantity of food in the stomach at the time, this stage occupies a longer or shorter period. Taken shortly after a good meal, the poison seems less rapid in action 
than when the stomach is empty. Presently come convulsions, and constant shrieking; then the limbs stick out and are perfectly stiff and rigid. Even at this stage the dog can often be saved if means are at hand. Never be without a bottle of syrup of chloral in the house ; it will keep indefinitely. First make the dog sick. Use sulphate of zinc in water, or weak mustard and warm water, and give plenty of this latter. The best way is by putting it in a phial, and running it down the throat by way of a pouch of lower lip drawn out from the teeth at the angle of the mouth. As soon as the patient has been sick, give a teaspoonful of the syrup of chloral in water. This is the antidote to strychnine. If you cannot wait to make the patient sick, give the chloral at once-but give it : and the dose may be repeated every two hours until the convulsions cease. For a tiny pup or dog under $5 \mathrm{lbs}$. the dose may be halved. Recovery from strychnine is very rapid, and it leaves, as a rule, no ill effects, though there is a widespread belief, and a mistaken one, that it subsequently affects the kidneys.

All the other kinds of poison dogs are likely to get or be given work as irritants, and these need veterinary diagnosis. Salt, I may here remark, is so violent and irritating a purgative to the dog that it is next door to a poison, and the effects of castor oil in his intestine are not so very far behind. Constant drugging is a thing as much to be avoided in dogs as in their owners, and I cannot too strongly deprecate the foolish practice -foolish or worse-of giving doses of castor oil after shows, or as so-called prophylactics-preventives of illness. If a dog has been much confined at a show, and is likely to be irregular in consequence, a little pure olive oil with his dinner (not the nut oil often sold by grocers as olive oil) will do no harm, although a dinner of oatmeal gruel or boiled sheep's liver would be much more sensible and act better; if he seems well and lively, leave him alone. Some people actually go the length of dosing their puppies with castor oil at in- 
tervals, for no reason that I can ascertain beyond a vague idea that it " clears the system." So it does-of strength and the healthy mucoid secretion of the intestine, without which natural functions cannot be properly performed. Syrup of buck-thorn, or cascara sagrada, is another medicine that should never be given to small dogs : it is far too irritating and severe. When we have such excellent aperients as olive oil, magnesia, and rhubarb among drugs, and boiled sheep's liver among meats, we want no semi-poisonous irritant and violent drugs like castor oil, which, in the end, produce the very condition they were supposed to cure, and by pulling down the system, open the door to illness.

Fits.-Of these, epileptic fits are the most dangerous and by far the least common. A dog suffering from epilepsy which is thoroughly established, is practically incurable, in the present state of canine medical science. Later, perhaps, the Röntgen rays may be beneficially applied to this disease in dogs, as in human beings. In a popular manual it is scarcely necessary to go further into the subject than to say that epilepsy need not be suspected unless the convulsive attacks are more or less recurrent, and so frequent as to exhaust the animal. Not until we have tried such treatment as an amateur can safely give, which is quite enough to cure ordinary teething or suckling fits due merely to some reflex irritation affecting the brain, and found it fail, need we fear epilepsy; and when we do fear it with any reason, skilled advice and diagnosis is absolutely needful, since the case must be watched and treated on its merits.

Suckling fits are exceedingly common among small, highly-organised, and sensitive bitches. They generally begin about the end of the second week of nursing puppies, and do not seem to be in any way caused by overstrain; that is, a small female suckling five puppies is not more likely to suffer from these fits than one only 
bringing up a brace. Their exact cause is difficult to determine, since very healthy, well-fed animals may have them in common with those that are weak and miserable from under-feeding (which in this case is synonymous with feeding on a non-meat diet) or kennel life.

Whatever the cause, the symptoms are always easy to recognise. The bitch first loses interest in her litter, though her milk-supply is seldom, if ever, lessened. She twitches, and her eyes look dull and filmy, or glassy and staring. She wanders restlessly about, and sometimes pants in the same way as she did when expecting her confinement. Now is the time to intervene, and give one teaspoonful of syrup of chloral with an equal quantity of water. If this is not done, the attack will proceed to staggering, shrieking, and more or less violent convulsions. The administration of the chloral generally causes the symptoms to subside gradually; but should the patient be no better in two hours, repeat the dose, and if giving bromide of potassium in 5-gr. doses twice or three times a day, immediately after food, does not keep her right, she must go on taking the chloral.

Neither chloral nor bromide affects the milk; if any of it passes therein, the quantity is so very minute as to make no difference to the puppies. It is not at all necessary to take the bitch away from her litter; in fact, it is better to let her go on feeding them. Some will wish to leave their babies, and these should be taken to them and shut in with them, four times a day, and during the night. If she is thoroughly well fed, it never does the bitch any harm to bring up her family, and it would be a very great pity for the puppies to be lost when it is not necessary. But it is exceedingly important that she should be kept in a state of hyper-nutrition -that is, that she should have as much good, underdone meat as she can digest. Bromides are lowering, and besides this, the state of the nerves demands the highest possible feeding. It may be expensive to feed 
a "fitty" bitch on good beefsteak or roast mutton four times a day, giving her a sponge cake the last thing at night and a little milk, or, what is much better and more digestible, a raw new-laid egg or raw fresh cream, in the early morning ; but it is, on the whole, a cheap way of saving a litter of valuable pups. If there are a large number of pups, some may be given to a fostermother ; but as a rule these are difficult to get, and not often satisfactory. Bromides should always be given immediately after food ; on no account when the stomach is empty. Chloral may be given at any time when there is a necessity for it. The 5-gr. bromide tabloids obtainable at any chemist's are very useful; it is unnecessary to dissolve them in water for dogs, but, as before stated, they must be given with or directly after food.

Teething fits should be treated, as far as medicine goes, exactly as suckling fits. Just as a badly-reared, non-meat-fed bitch who, by reason of an anæmic habit, harbours worms, is a poor subject for the latter trouble, so is a puppy that has been brought up on milky slops and large, wet messes of oatmeal and bread and milk, and thus has a weakened digestion, very likely to suffer badly from fits that in a strong young dog would pass off with small trouble. There is usually some warning of teething fits, as staring eyes, etc. ; but sometimes, and especially if a puppy of from six to ten months has been much excited, taken out walking on a hot day, allowed to play in the sun, or dragged unwillingly on a lead, they come on very suddenly. While out in het sun, the dog may suddenly give a shriek and begin to run with all his might, taking no notice of calls. As a general rule, he has the sense to run home, unless some officious person on the way imagines him mad and acts as silly people do under such circumstances.

If it is possible to catch the runaway, he should have his head covered to keep the light out of his eyes, and be taken home as quickly and quietly as possible to be shut 
in some cool and perfectly dark place until the fit passes off sufficiently to give him a dose of chloral. Afterwards he should have a diet of minced, underdone meat, with bromide of potassium to follow, for a day or two. A plunge into cold water will often stop a fit like this, but is too heroic a remedy to be safe unless the circumstances are very urgent. Cold sponging to the head is good, and quiet and darkness are essential. Some times teething fits go on increasing in frequency and severity until they merge into epilepsy, and the dog is lost. This is occasionally caused by allowing a very young, highly nervous, and excitable dog to be with others of the opposite sex, when these should be in seclusion.

Fits, very much like mild teething fits, are not uncommon in run-down dogs suffering from anæmia and the likely corollary, worms. These are often very transient, and a course of tonic treatment, with rest from excite. ment, and good feeding, will banish them.

\section{CHAPTER IX}

CLUB STANI)ARDS, DESCRIPTIONS AND POINTS OF VARIOUS TOY BREEDS

Pomeranians.-These are now divided into Pomeranians (over 7 lbs.) and Pomeranians Miniature, and the Committee of the Kennel Club have laid down the following standard, applying from June I, I909:

The Pomeranian.-Appearance.-The Pomeranian in build and appearance should be a compact, short-coupled dog, well-knit in frame. His head and face should be fox-like, with small erect ears that appear sensible to every sound. He should exhibit great intelligence in his expression, docility in his disposition, and activity and buoyancy in his deportment. In weight and size the Pomeranian varies considerably. He must be over 
7 lbs., but preferably he should weigh about ro to I4 $1 \mathrm{bs}$. Head. - The head should be somewhat foxy in outline or wedge-shaped, the skull being flat, large in proportion to the muzzle, which should finish rather fine, and be free from lippiness. The teeth should be level, and on no account undershot. The hair on the head and face must be smooth and short-coated.

The Pomeranian Miniature-Appearance.-The Pomeranian Miniature in build and appearance should be a compact, short-coupled dog. His head and face should be like a miniature fox, with small, erect, and very mobile ears, pricked and brought well together, and in no case lop-eared. He should be full of life, intelligent in expression, and docile in disposition. The Pomeranian Miniature should preferably weigh about 3 to $5 \mathrm{lbs}$., but must not exceed 7 lbs. Dogs above $7 \mathrm{lbs}$. must be registered as Pomeranians. Dogs below 7 lbs. in weight must, at twelve months of age or after, be registered or re-registered as Pomeranians Miniature, and being so registered or re-registered, can never compete in classes for Pomeranians. Head.-The head should be wedge-shaped and rather foxy in outline, but the skull may be rounder than the Pomeranian.

Standard and Scale of Points as laid down by the Pomeranian Club.-Secretary, G. M. Hicks, Esq., Granville House, Blackheath, London, S.E.* Appearance.-The Pomeranian in build and appearance should be a compact, short-coupled dog, well-knit in frame. His head and face should be fox-like, with small, erect ears, that appear sensible to every sound; he should exhibit great intelligence in his expression, docility in his disposition, and activity and buoyancy in his deportments. - I5 points. Head.-Somewhat foxy in outline, or wedge-shaped, the skull being slightly flat (although in

* In most cases the names of the Secretaries of the various clubs are given, but it must be remembered that an annual re-election takes place. 
the toy varieties the skull may be rather rounder), large in proportion to the muzzle, which should finish rather fine, and be free from lippiness. The teeth should be level, and on no account undershot. The head in its profile may exhibit a little " stop," which, however, must not be too pronounced, and the hair on head and face must be smooth or short-coated.-5 points. Eyes.Should be medium in size, rather oblique in shape, not set too wide apart, bright and dark in colour, showing great intelligence and docility of temper. In a white dog black rims round the eyes are preferable. -5 points. Ears. - Should be small, and carried perfectly erect, or pricked like those of a fox, and, like the head, should be covered with soft, short hair. No plucking or trimming is allowable.-5 points. Nose.-In black-and-tan, or white dogs, the nose should be black ; in other coloured Pomeranians it may more often be brown or liver coloure 1; but in all cases the nose must be self rot parti-coloured, and never white. -5 points. Neck ant Shoulders.-The neck, if anything, should be rather short, well set in and lion-like, covered with a profuse mane and frill of long, straight, glossy hair, sweeping from under the jaw, and covering the whole of the front part of the shoulders and chest, as well as flowing on the top of the shoulders. The shoulders must be tolerably clean and laid well back.-5 points. Body.-The back must be short, and the body compact, being well ribbed up, and the barrel well rounded. The chest must be fairly deep, and not too wide.-ro points. Legs.-The forelegs must be perfectly straight, of medium length-not such as would be termed either "leggy" or "low on leg" - but in due proportion in length and strength to a well-balanced frame, and the forelegs and thighs must be well feathered, the feet small and compact in shape. No trimming is allowable. -5 points. Coat. - Properly speaking, there should be two coats, an under and an over coat - the one a soft, fluffy under coat, the other a long, perfectly straight and glistening coat, covering the wholc of the body, being very abundant 
round the neck and forepart of the shoulders and chest, where it should form a frill of long, flowing hair, extending over the shoulders, as previously described. The hindquarters, like those of a collie, should be similarly clad with long hair or feathering from the top of the rump to the hocks. The hair on the tail must be profuse and flowing over the back. -25 points. Tail. -The tail is a characteristic of the breed, and should be well twisted right up from the root tightly over the back, or lying flat on the back, slightly on either side, and profusely covered with long hair, spreading out and flowing over the back. - Io points. Colour. - The following colours are admissible: White, black, blue, brown, black-and-tan, fawn, sable, red, and parti-colours. The white must be quite free from lemon or any colour, and the blacks, blues, browns, black-and-tan, and reds free from white. A few white hairs in any of the selfco'ours shall not absolutely disqualify, but should carry great weight against the dog. In parti-coloured dogs, the colours should be evenly distributed on the body. Whole-coloured dogs with a white foot or feet, leg or legs, are decidedly objectionable, and should be discouraged, and cannot compete as whole-coloured specimens. In mixed classes-i.e., where whole-coloured and parti-coloured Pomeranians compete together-the preference should, if in other points they are equal, be given to the whole - coloured specimens. - Io points. Total-roo points.

Also catered for by the North of England Pomeranian Club. Secretary, J. Tweedale, Valley House, Oversley Ford, Wilmslow ; and the Midland Counties Pomeranian Club. Hon. Secretary, Mrs. E. Parker, Meadowland, Uttoxeter Road, Derby.

Toy Spaniels (English).-Points as defined by the Toy Spaniel Club. Hon. Secretary, Miss M. Hall, Chalk Hill House, Norwich. Head.--Should be well domed, and in good specimens is absolutely semi-globular, sometimes even extending beyond the half-circle, and 
absolutely projecting over the eyes, so as nearly to meet the upturned nose. Eyes.-The eyes are set wide apart, with the eyelids square to the line of the face -not oblique or fox-like. The eyes themselves are large, so as to be generally considered black; their enormous pupils, which are absolutely of that colour, increasing the description. From their large size, there is always a certain amount of weeping shown at the inner angles; this is owing to a defect in the lachrymal duct. Stop.-The "stop" or hollow between the eyes, is well marked, as in the bulldog, or even more so; some good specimens exhibiting a hollow deep enough to bury a small marble. Nose.-The nose must be short and well turned up between the eyes, and without any indication of artificial displacement afforded by a deviation to either side. The colour of the end should be black, and it should be both deep and wide, with open nostrils. Jaw. - The lower jaw must be wide between its branches, leaving plenty of space for the tongue and for the attachment of the lower lips, which should completely conceal the teeth. It should also be turned up or " finished," so as to allow of its meeting the end of the upper jaw, turned up in a similar way, as above described. Ears.-The ears must be long, so as to approach the ground. In an average-sized dog they measure 20 ins. from tip to tip, and some reach 22 ins., or even a trifle more. They should be set low on the head, and be heavily feathered. In this respect the King Charles is expected to exceed the Blenheim, and his ears occasionally extend to 24 ins. Size.-The most desirable size is from 7 lbs. to Io lbs. Shape.-In compactness of shape these spaniels almost rival the pug, but the length of coat adds greatly to the apparent bulk, as the body, when the coat is wetted, looks small in comparison with that dog. Still, it ought to be decidedly " cobby," with strong, stout legs, broad back, and wide chest. The symmetry of the toy spaniel is of importance, but it is seldom that there is any defect in this respect. Coat.-The coat should be long, silky, 
soft, and wavy, but not curly. In the Blenheim there should be a profuse mane, extending well down in the front of the chest. The feather should be well displayed on the ears and feet, where it is so long as to give the appearance of their being webbed. It is also carried well up the backs of the legs. In the King Charles the feather on the ears is very long and profuse, exceeding that of the Blenheim by an inch or more. The feather on the tail (which is cut to the length of about $3 \frac{1}{2}$ ins. to 4 ins.) should be silky, and from 5 ins. to 6 ins. in length, constituting a marked "flag" of a square shape, and not carried above the level of the back. Colour.-The colour varies with the breed. The King Charles is a iich, glossy black, and deep tan; tan spots over the eyes and on cheeks, and the usual markings on the legs are also required. The Ruby Spaniel is a rich chestnut red. The presence of a few white hairs intermixed with the black on the chest of a King Charles, or intermixed with the red on the chest of a Ruby Spaniel, shall carry very great weight against a dog, but shall not in itself absolutely disqualify; but a white patch on the chest, or white on any other part of a King Charles or Ruby Spaniel shall be a disqualification. The Blenheim must not on any account be whole-coloured, but should have a ground of pure pearly white, with bright, rich chestnut or ruby-red marking evenly distributed in large patches.

The ears and cheeks should be red, with a blaze of white extending from the nose up to the forehead, and ending between the ears in a crescentive curve. In the centre of this blaze there should be a clear "spot" of red of the size of a sixpence. The tricolour, or Charles the First Spaniel, should have the tan of the King Charles, with markings like the Blenheim in black instead of red on a pearly-white ground. The ears and under the tail should also be lined with tan. The tricolour has no spot, that beauty being peculiarly the property of the Blenheim.

The only name by which the tricolour, or black, 
white, and $\tan$, in future shall be recognised is " Prince Charles."

That in future the all-red toy spaniel be known by the name of "Ruby Spaniel." The colour of the nose to be black. The points of the "Ruby" to be the same as those of the "King Charles," differing only in colour.

\section{Scale of Points.}

King Charles, Prince Charles, and Ruby Spaniels.

Symmetry, condition, and size ....... 20

Head

Stop

Muzzle

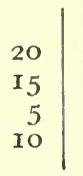

Eyes

Ears

I5

Coat and feathering . I5

Colour .......... ro

TOTAL $\overline{\text { IOO }}$

Blenheim.

Symmetry, condition, and size $\ldots \ldots \ldots$ I. I 5

Head $\ldots \ldots \ldots \ldots$ I 15

Stop .......... 5

Muzzle ........ ro

Eyes ........... Io
Ears ............ ro

Coat and feathering . I 5

Colour and markings 15

Spot ........... 5

The Toy Trawler Spaniel.-This little dog, having had some classes given for it at shows, deserves notice, and its standard and scale of points are appended, together with some remarks made upon it by a lady who has introduced it, and whose kennel of beautiful Toy Spaniels of all breeds is well known. Points.-Head small and light, with very pointed, rather short, nose, fine and tapery, with a very slight curve upwards of tip of nose. A curve downwards (as in the Borzoi) should be an absolute disqualification. The "stop" well marked, and the skull rather raised, but flat on the top, not dome-shaped. Muzzle just finished, not overshot. Long ears, set high, and carried pricked forwards, framing the face. Large dark eyes, set wide apart, and 
showing the white when turned. They must be set perfectly straight, not obliquely, in the head. Whatever colour the dog may be, the nose and lips must be black. Neck arched. Back broad and shcrt. Tail set on a level with the back, and carried gaily, though not straight up in the air, or curled over the back like a Pomeranian. It should be docked to about 4 or 5 inches, and well furnished with long feathering. General carriage very smart and gay. Legs reasonably short, and perfectly straight, bone light, though strong. Build square, sturdy, and compact, but never heavy. The action should be smart and prancing, coat very curly, but not woolly. It should be rather silky in texture, and very glossy. Liberal feathering, waistcoat, and breechings. Shape is all important ; colour a secondary matter. Best colour a brilliant black, with white waistcoat. Next, red with white waistcoat, black and white, and red and white. Best size from II to I3 inches at shoulder. Any tendency to weediness should be carefully avoided, and the height at shoulders should just about equal the length from top of shoulders to root of tail. The size should not be judged by weight, but by height, as they should weigh heavily for their size. A dog about I3 inches high should weigh about I5 lbs. Very small specimens-i.e., under 9 inches high-are only desirable if the type, soundness, compactness, and sturdiness are unimpaired. Feet close, firm, and hard. They and the lower part of the legs should not be too heavily feathered. The expression of face should be very alert, and very sweet. The dogs should be very bold and courageous. Timidity is a great fault, and should tell against them in the ring. They are excellent ratters and rabbiters. As to proportion of head, if the total length of head be about 6 inches, the ears should be set about 4 inches apart. The whole head, seen from a bird's-eye point of view, should be triangle, with the tip of nose as apex. General appearance should be that of an exquisitely pretty little sporting dog, very strong, and exceedingly smart and compact. 
They must not be confounded with Cockers, being a totally different type.

Scale of Points.

General appearance, including condition and smartness ....

Coat ..............

Head and expression

Eyes .............

Curve and proportion of muzzle ........

Set on of ears ......

Legs and feet ......

Colour ........... 5
Action and soundness of $\operatorname{limb} \ldots \ldots \ldots$, Io

Size ........... 5

Compactness, levelness of back, and set of tail ....... ro

Boldness and alertness ......... 8 Soundness of teeth .. 3

Total ioo

\section{Points that Should Disqualify.}

I. A flesh-coloured nose.

2. A downward curve of muzzle.

3. No "stop."

4. Hanging lips.
5. Crooked forelegs.

6. Light-coloured eyes.

7. Slanting eyes.

8. A very long body.

9. Bad action.

Points that are Very Undesirable.

I. Timidity.

2. A straight coat.

3. Low set ears.

4. Exaggeratedly short or long legs.

5. Sluggishness:
6. Exaggeration of any kind.

7. Drooping tail.

8. Showing teeth or tongue.

9. An "apple" head.

\section{Measurements of a Perfect Specimen.}

Breadth of skull at eyes from each outside corner of eyes across head ...... 5

Length of skull ..... 4

Length of nose ..... $2 \frac{1}{4}$

Circumference of skull Io $\frac{1}{2}$

Circumference of muzzle under eyes Space between eyes.. Space between ears when not pricked .

$6 \frac{3}{4}$
$1 \frac{3}{8}$

Length of ears (leather) Inches

Height at shoulders .. I3

Length from top of shoulders to root of tail ..............

Length of forelegs to elbow ........... $\quad 7 \frac{1}{2}$

Breadth at shoulders . 6

Breadth at quarters .. 6

Girth .......... I9

Feathering on tail flag 6

Waistcoat feathering . 4 
The origin of the breed is unknown, but it is supposed to be descended from the original curly King Charles Spaniel (see Mr. Watson's "Book of the Dog") and the oldfashioned curly Sussex Spaniel, now extinct. There is no certainty in this. The breed exists in Italy and Holland.

Toy Spaniels also have the Northern Toy Spaniel Club. Secretary, Mrs. E. A. Furnival, Eastwood, Mauldeth Road, Heaton Mersey, Manchester.

Griffons Bruxellois. - Points as defined by the Griffon Bruxellois Club. Hon. Secretary, Miss L. Feilding, 48, Grosvenor Gardens, London, S.W. General Appearance.-A lady's little dog, intelligent, sprightly, robust, of compact appearance, reminding one of a cob, and captivating the attention by a quasi-human expression. Head.-Rounded, and covered with coarse, rough hairs, somewhat longer round the eyes and on the nose, lips, and cheeks. Ears.-Erect when clipped, semi-erect when not clipped. Eyes.-Very large without being watery, round, nearly black; eyelids edged with black; eyelashes long and black, leaving the eye they encircle perfectly uncovered. Nose.-Always black, short, surrounded with hair converging upwards and going to meet that which surrounds the eyes; the break (or stop in the nose) pronounced, but not exaggerated. Lips.-Edged with black, furnished with moustache; a little black in the moustache is not a fault. Chin.-Prominent, without showing the teeth, and edged by a small beard. Chest.-Rather wide. Legs. - As straight as possible, of medium length. Tail. -Upward, and cut to the two-thirds. Colour.-Red. Texture of Coat.-Harsh and wiry, rather long. Weight. - Light weight $5 \mathrm{lbs}$. maximum, and heavy weight 9 lbs. the maximum. Faults.-Brown nose, pale-coloured eyes, silky tuft on the head, white spot on the chest or paw.

\section{Scale of Points.}

Hard coat ........

Reddish colour..... I

Eyes

Nose and muzzle....

Ears
I 5
Legs and body......

Height and size

General appearance .

5

IO

\section{Total 60}


The Brussels Griffon Club of London (Secretary, Miss A. F. Hall, 2, Park Place Villas, Maida Hill, London, W.) offers practically the same standard, but makes a brown nose, white hairs, and a hanging tongue disqualify, while as faults it cites light eyes, silky hair on head, brown nails, and teeth showing; and its description of the typical coat is as follows:-Texture of coat harsh and wiry, irregular, rather long and thick.

Schipperkes.-The description of the Schipperke adopted at a general meeting of the Belgian Schipperke Club, June I9th, I888, has been adopted by the St. Hubert Schipperke Club, and is copyright. The Schipperke Club, England, advances the following scale of points, and the Secretary is G. H. Killick, Esq., Moor House, Chorley, Lancashire.

Head.-Foxy in type ; skull should not be round, but broad, and with little "stop." The muzzle should be moderate in length ; fine, but not weak; should be well. filled out under the eyes. Nose. - Black and small. Eyes.-Dark brown, small, more oval than round, and not full ; bright and full of expression. Ears.-Shape : Of moderate length, not too broad at the base, tapering to a point. Carriage: Stiffly erect, and, when in that position, the inside edge to form as near as possible a right angle with the skull, and strong enough not to be bent otherwise than lengthways. Teeth.-Strong and level. Neck.-Strong and full, rather short, set broad on the shoulders, and slightly arched. Shoulders. -Muscular and sloping. Chest.-Broad and deep in brisket. Back.-Short, straight, and strong. Loins.Powerful, well drawn up from the brisket. Forelegs.Perfectly straight, well under the body, with bone in proportion to the body. Hindlegs.-Strong, muscular ; hocks well let down. Feet.-Small, catlike, and standing well on the toes. Nails.-Black. Hindquarters.Fine compared to the foreparts; muscular and welldeveloped thighs; tailless; rump well rounded. Coat. -Black, abundant, dense, and harsh, smooth on the 
head, ears, and legs; lying close on the back and sides, but erect and thick round the neck, forming a mane and frill, and well feathered on back of thighs. Weight.-About I2 lbs. General Appearance.-A small, cobby animal, with sharp expression, intensely lively, presenting the appearance of being always on the alert. Disqualifying Points.-Drop or semi-erect ears. Faults. -White hairs are objected to, but are not disqualifying.

\section{Relative Value of Points.}

Head, nose, eyes, and teeth .......... 20

Ears ............ Io

Neck, shoulders, and chest .......... ro

Back and loins ..... 5

Forelegs ......... 5
Hindlegs $\ldots . . . \ldots 5$

Feet $\ldots \ldots \ldots \ldots, 5$

Hindquarters ..... ro

Coat and colour .... 20

General appearance . Io

Total IOO

The St. Hubert Schipperke Club standard is practically identical with that of the Schipperke Club, England, the only variation being as regards the weight limits, which this club, however, also fixes at a maximum of I2 lbs. for small-sized dogs, while it allots 30 points to coat and colour, and none to general appearance. They also have the Northern Schipperke Club. Hon. Secretary, T. W. Markland, Ingersley, Links Gate, St. Anne's-on-the-Sea.

Pugs.-Standard and acknowledged points :

THE STANDARD.

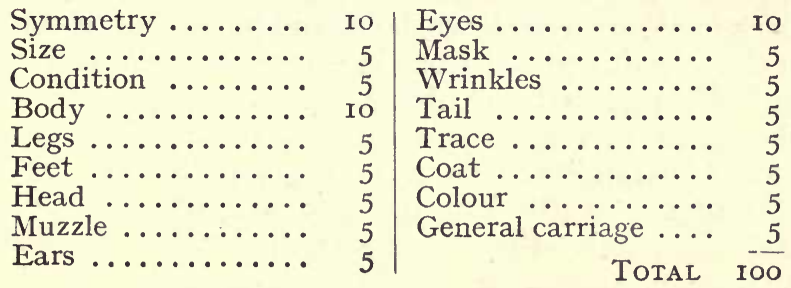




\section{Acknowledged Points.}

Symmetry.-Symmetry and general appearance, decidedly square and cobby. A lean, leggy pug and a dog with short legs and a long body are equally objectionable. Size and Condition.- - The pug should be multum in parvo, but this condensation (if the word may be used) should be shown by compactness of form, wellknit proportions, and hardness of developed muscle. Weight from I3 lbs. to I7 lbs., dog or bitch. Body.Short and cobby, wide in chest, and well ribbed up. Legs.-Very strong, straight, of moderate length, and

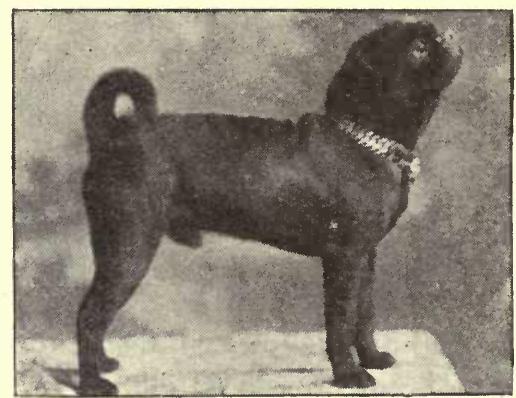

BLACK PCG.

"Larchmoor Peter Pan;' owned b" Mrs. Lyle.

well under. Feet.-Neither so long as the foot of the hare nor so round as that of the cat; well split-up toes, and the nail black. Muzzle.--Short, blunt, square, but not up-faced. Head.-Large, massive, round, not apple-headed, with no indentation of the skull. Eyes.Dark in colour, very large, bold, and prominent, globular in shape, soft and solicitous in expression, very lustrous, and, when excited, full of fire. Ear.-Thin, small, soft, like black velvet. There are two kinds, the "rose" and "button." Preference is given to the latter. Markings.-Clearly defined. The muzzle or mask, ears, moles on cheeks, thumb-mark or diamond on forehead, back-trace, should be as black as possible. Mask.- 
The mask should be black. The more intense and well-defined it is the better. Wrinkles. - Large and deep. Trace.-A black line extending from the occiput to the tail. Tail.-Curled tightly as possible over the hip. The double curl is perfection. Coat.-Fine, smooth, soft, short, and glossy, neither hard nor woolly. Colour. - Silver, or apricot fawn. Each should be decided, to make the contrast complete between the colour and the mask and trace. N.B.-The points of black pugs, except as to colour, are the same as those for fawns. The London and Provincial Pug Club. Secretary, J. Fabian, 460, Camden Road, London, N.

Toy Bulldogs. - Points of Toy Bulldogs.-The general appearance of the toy bulldog must, as nearly as possible, resemble that of the big bulldog. The skull should be large, forehead flat, the skin about it well wrinkled, the "stop" broad and deep, extending up the middle of the forehead. Eyes of moderate size, situated low down on the skull, and as wide apart as possible. Ears to be "rose," if possible ; "tulip " ears are allowable, but not to be encouraged ; "button," or terrier-like ears are a decided fault. Face to be as short as possible, nose jet black, deeply set back, almost between the eyes. Muzzle to be short, broad, and turned upwards. The lower jaw should project considerably in front of the upper and turn up. Teeth not to be shown. Neck to be short, with much loose skin about it. "Frogginess" is objectionable. Chest to be very wide, round, and deep. Back short and strong, narrow towards the loins, and broad at the shoulder. A roach back is desirable. Tail to be short, and not carried above the back. Forelegs to be short in proportion to the hindlegs. Hindquarters much lighter in proportion than forequarters. The most desirable weight is below 20 lbs., and dogs and bitches that exceed $22 \mathrm{lbs}$. should be disqualified. The Miniature Bulldog Club. Secretary, Miss A. Bruce, 42, Hill Street, Berkeley Square, London, W. 


\section{Scale of Points.}

General appearance and character .... Io

Head ........... I $_{5}$

Ears .......... I5

Body ......... Io
Size and weight ... 20

Tail .......... 5

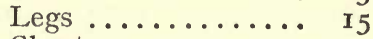

Chest ......... ro

Total $\overline{100}$

Description and Points of the French Toy BullDOG.-General Appearance.-The French bulldog ought to have the appearance of an active, intelligent, and

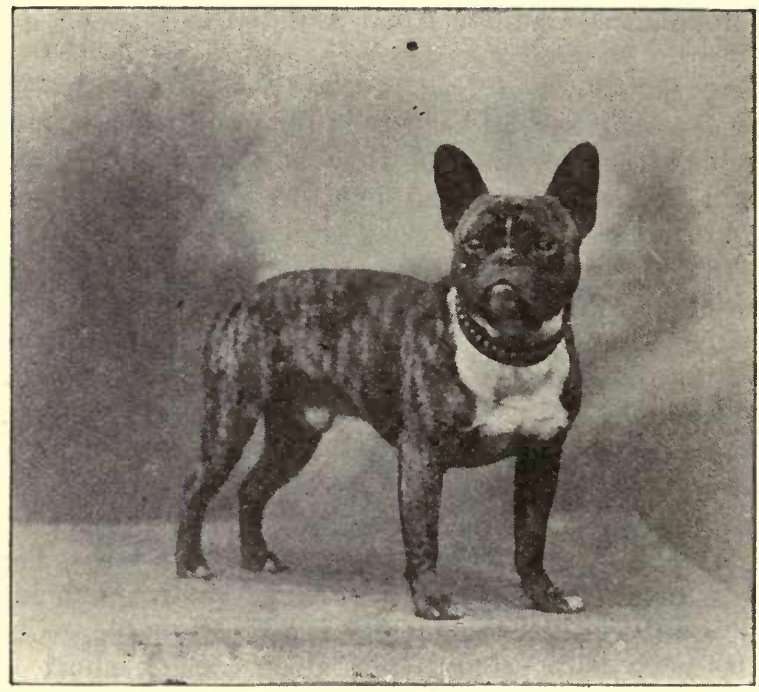

FRENCH TOY BULLDOG.

"Barkston Billie," ozened by Mrs. Townsend Green.

very muscular dog, of cobby build, and heavy in bone for its size. Head is of great importance, large and square. Forehead nearly flat, the muscles of the cheek well developed, but not prominent. The "stop" should be as deep as possible. The skin of the head should not be tight, and the forehead should be well. 
wrinkled. The muzzle should be short, broad, turn upwards, and be very deep. The lower jaw should project considerably in front of upper, and should turn up, but should not show the teeth. The eyes should be of moderate size and of dark colour. No white should be visible when the dog is looking straight in front of him. They should be placed low down and wide apart. The nose must be black and large. Ears.-Bat ears ought to be of a medium size, large at the base and rounded at the tips. They should be placed high on the head and carried straight. The orifice of the ear looks forward, and the skin should be fine and soft to the touch. The neck should be thick, short, and well arched. The body.-The chest should be wide and well down between the legs, and the ribs well sprung. The body short and muscular, and well cut up. The back should be broad at the shoulder, tapering towards the loins, preferably well roached. The tail should be set on low, and be short, thick at the root, tapering to a point, and should not be carried above the level of the back. Legs. - The forelegs should be short, straight, and muscular. The hindquarters, though strong, should be lighter in proportion to the forequarters. Hocks well let down. Feet should be compact and strong. Coat should be of a medium density : black in colour is very undesirable. Their Club is the Bouledogue Français Society. Secretary, F. Everard, Ir, Milk Street, London, E.C.

\section{Scale of Points.}

General appearance and character ..... Skull

Under jaw (special points for) ....... Io Weight* ......... 20
Body $\ldots \ldots \ldots \ldots$. I 5

Tail ........... 5

Ears (bat) ....... Io

Legs ............ 5

Chest .......... 5

TOTAL $\overline{\text { IOO }}$ 22 lbs.

* No dog to win the maximum of points unless under

Weights.-When three classes are provided, weights shall 
Yorkshire Terriers.-Points of the Yorkshire Terrier, as laid down by the Yorkshire Terrier Club. Secretary, Mr. F. W. Randall, "The Clone," Hampton-onThames. General Appearance.- Should be that of a long-coated pet dog, the coat hanging quite straight and evenly down each side, a parting extending from the nose to the end of the tail. The animal should be very compact and neat, the carriage being very upright, and having an important air. Although the frame is hidden beneath a mantle of hair, the general outline should be such as to suggest the existence of a vigorous and wellproportioned body. Head. - Should be rather small and flat, not too prominent or round in the skull, nor too long in the muzzle, with a perfectly black nose. The fall on the head to be long, of a rich golden tan, deeper in colour at the sides of the head about the ear roots, and on the muzzle, where it should be very long. The hair on the chest a rich bright tan. On no account must the tan on the head extend on to the neck, nor must there be any sooty or dark hair intermingled with any of the tan. Eyes.-Medium, dark, and sparkling, having a sharp, intelligent expression, and placed so as to look directly forward. They should not be prominent, and the edge of the eyelids should be of a dark colour. Ears.-Small V-shaped, and carried semi-erect or erect, covered with short hair, colour to be of a very deep rich tan. Mouth.-Perfectly even, with teeth as sound as possible. An animal having lost any teeth through accident not a fault, providing the jaws are even. Body. - Very compact, and a good loin. Level on the top of the back. Coat.-The hair on body as long as possible, and perfectly straight (not wavy), glossy like silk, and of a fine silky texture. Colour, a dark steel blue (not

be as follows: (I) Under 20 lbs.; (2) $20 \mathrm{lbs}$. and under 24 lbs. ; (3) $24 \mathrm{lbs}$. and under $28 \mathrm{lbs}$.

When only two classes are provided, weights shall be as follows: (I) Under 24 lbs. ; (2) 24 lbs., not exceeding 28 lbs.

These weights are subject to alteration. 
silver blue) extending from the occiput (or back of skull) to the root of tail, and on no account mingied with fawn, bronze, or dark hairs. Legs.-Quite straight, well covered with hair of a rich golden tan, a few shades lighter at the ends than at the roots, not extending higher on the forelegs than the elbow, nor on the hindlegs than the stifle. Feet.-As round as possible, and the toe-nails biack. Tail.-Cut to medium length; with plenty of hair, darker blue in colour than the rest of the body, especially at the end of the tail, and carried a little higher than tho level of the back. Tan.-All tan hair should be darker at the roots than in the middle, shading to a still lighter $\tan$ at the tips. Weight.Three classes : $5 \mathrm{lbs}$. and under ; $7 \mathrm{lbs}$. and under, but over 5 lbs. ; over 7 lbs.

"Silver" Yorkshire.--Points identical with those of the Standard Yorkshire, as dèscribed above, except colouring, which should be as follows: Back.-Silver. Head.-Pale tan or straw colour. Muzzle and Legs.Light tan. Ears.-A shade dárker tan.

\section{Value of Points in Judging.}

Quantity and length of coat ...........

Quality and texture of coat ..........

Richness of tan on head and legs ....

Colour of hair on body $\ldots \ldots \ldots \ldots$. IJ

Head

I5
IO
I5
I 5
IO

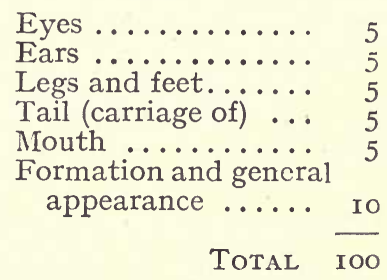

Italian Greyhounds. - The Italian Greyhound is somewhat fuller in proportion than the English Greyhound, and the nose is somewhat shorter. In other respects this beautiful dog follows the lines of its prototype as closely as possible, due allowance-being made for difference in size. The colour most prizad is a golden fawn, then cream, or blue fawn, followed by reds and whites; mixtures are not considered desirable. Coat 
should be very fine, soft, and glossy. The best size is that of a dog of about 8 lbs. weight.-From Rawdon Lee's “Modern Dogs." Hon. Secretary of Club, Mrs. Scarlett, Went House, West Malling, Kent.

Maltese.-This is probably the oldest of the toy dogs, having been highly prized by the ladies of ancient Greece, and doubtless of other nations at the same time. The coat is very long, stiaight, and silky (in first-rate specimens sweeping the ground), quite free from woolliness and from the slightest curl. Colour, pure white. Nose should be black, also roof of the mouth. Ears moderately long, the hair on them mingling with that on the neck. Tail short and well feathered, curled tightly over back. Size should not exceed 5 lbs. or 6 lbs., the smaller the better, other points being correct.-Rawdon Lee's " Modern Dogs." "They have the Maltese Club of London. Hon. Secretary, Arthur Stevenson, 52, Holloway Road, N.

Poodles.-Points of the perfect black poodle, as defined by the Poodle Club. Secretary, Mr. L. W. Crouch, The Orchard, Swanley Village, Kent. General Appearance:-That of a very active, intelligent, and elegant-looking: dog, well built, and carrying himself very proudly. Head.-Long, straight, and fine, the skull not broad, with a slight peak at the back. Muzzle.Long (but not snipy) and strong; not full in cheek ; teeth white, strong, and level ; gums black; lips black and not showing lippiness. Eyes.-Almond-shaped, very dark, full of fire and intelligence. Nose.-Black and sharp. Ears.-The leather long and wide, low set on, hanging close to the face. Neck.-Well proportioned and strong, to admit of the head being carried high and with dignity. Shoulders.-Strong and muscular, sloping well to the back. Chest.-Deep and moderately wide. Back.-Short, strong, and slightly hollowed, the loins broad and muscular, the ribs well sprung and braced up. Feet.-Rather small and of a good shape, the toes well arched, pads thick and hard. 
Legs. - Fore set straight from shoulder, with plenty of bone and muscle ; hindlegs very muscular and well bent, with the hocks well let down. Tail.-Set on rather high, well carried, never curled, or carried over back. Coat.-Very profuse, and of good, hard texture; if corded, hanging in tight, even cords; if non-corded,

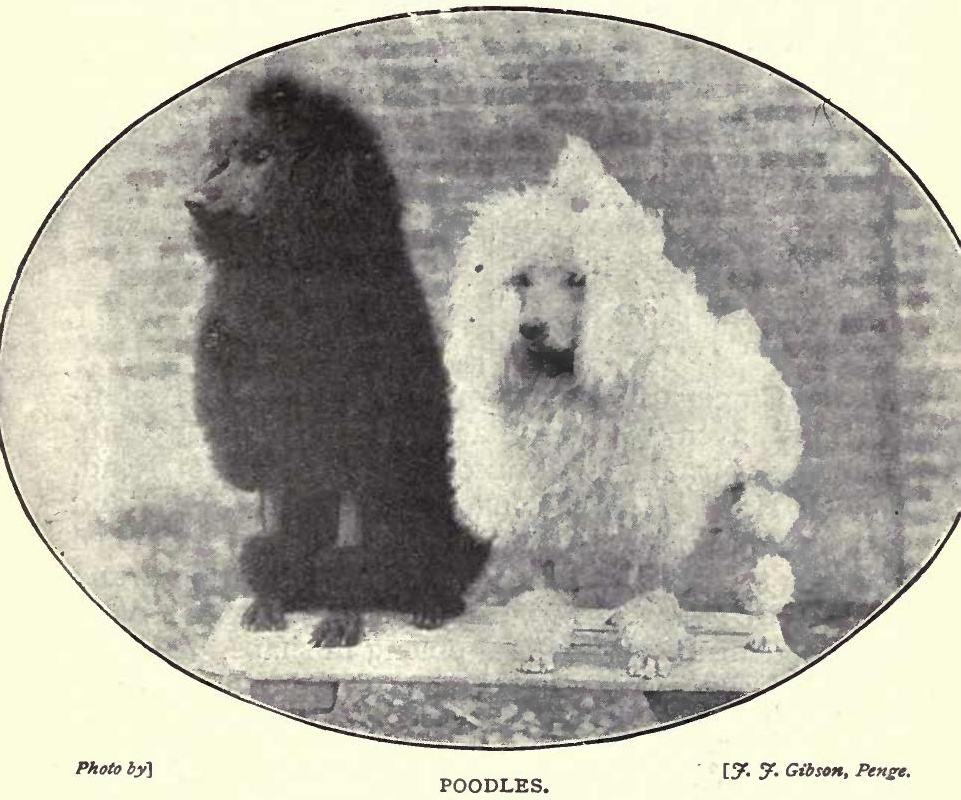

Champion "Orchard Admiral" and "L'Enfant Prodigue," owned by Mrs. Crouch.

very thick and strong, of even length, the curls close and thick, without knots or cords. Colours.-All black, all white, all red, all blue. The white poodle should have dark eyes, black or very dark liver nose, lips, and toe-nails. The red poodle should have dark amber eyes, dark liver nose, lips, and toe-nails. The blue poodle should be of even colour, and have dark eyes, 
lips, and toe-nails. All the other points of white, red, and blue poodles should be the same as in the perfect black poodle. N.B.-It is strongly recommended that only one-third of the body be clipped or shaved, and that the hair on the forehead be left on.

Also catered for by the Curly Poodle Club, Hon. Secretary, Miss F. Brunker, Whippendell House, King's Langley, Herts.

\section{Value of Points.}

General appearance and movement ...

Head and ears

Eyes and expression

Neck and shoulders . Shape of body, loin, back, and carriage of stern
Legs and feet

Coat, colour, and

$\mathrm{I}_{5}$

$\mathbf{r}_{5}$

Io

ro

I5
IO ture of coat

Bone, muscle, and condition ........

Total IOO

The Black-and-Tan Terrier.-Points and standard, as given by the Black-and-Tan Terrier Club. Secretary, Mr. S. J. Atkinson, I84, Adelaide Road, London, N.W. Head.-Long, flat, and narrow, level and wedge-shaped, without showing cheek muscles, well filled up under the eyes, with tapering, tightly-lipped jaws and ievel teeth. Eyes.-Very small, sparkling, and dark, set fairly close together, and oblong in shape. Nose.Black. Ears.-Small and V-shaped, hanging close to the head above the eye. Neck and Shoulders.-The neck should be fairly long, and tapering from the shoulders to the head, with sloping shoulders, the neck being free from throatiness, and slightly arched at the occiput. Chest.-Narrow, but deep. Body.-Moderately short, and curving upwards at the loin; ribs well sprung; back slightly arched at the loin, and falling again at the joining of the tail to the same height as the shoulders. Legs.-Must be quite straight, set on well under the dog, and of fair length. Feet.-More inclined to be cat than hare-footed. Tail.-Moderate length, and set on where the arch of the back ends, thick where it joins the body, tapering to a point, 
and not carried higher than the back. Coat.-Close, smooth, short, and glossy. Colour.-Jet black and rich mahogany tan, distributed over the body as follows: On the head the muzzle is tanned th the noso', which. with the nasal bone, is jet. black; there is also a bright tan spot on each cheek and above each eys; the under jaw and throat are tannea, and the hall inside the ear is of the same colour. The forelegs tanned up to the knee, with black lines (pencil marks) up each toe, and a black mark (thumb mark) above the foot. Inside the hindlegs tanned, but divided with black at the hock joint, and under the tail also tanned, and so is the vent, but only sufficiently to be easily covered by the tail ; also slightly tanned on each side of chest. Tan outside of hindlegs, commonly called "breeching," a serious defect. In all cases the black should not run into the tan, or vice versa, but the division between the two colours should be well defined. General Appearance.A terrier, calculated to take his own part in the rat-pit, and not of the whippet type. Weight (for toys).-Not exceeding $7 \mathrm{lbs}$.

\section{SCAlE of Points.}

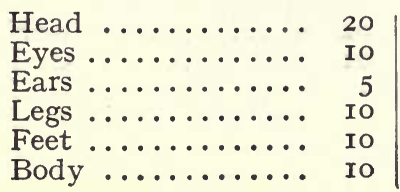

$$
\begin{aligned}
& \text { Tail .............. } \\
& \begin{array}{c}
\text { Colour and markings } \\
\text { General appearance } \\
\text { (including terrier } \\
\text { quality) } \ldots . . . . .
\end{array} \\
& \text { ToraL }
\end{aligned}
$$

Japanese and Pekingese Spaniels.-Points of the Japanese spaniel, as set forth by the Japanese and Pekingese Club. This Club is now divided into the Japanese Chin Club and the Pekingese Club, the Secretary of both being Mr. E. T. Cox, 65 and 66, Chancery Lane, London, E.C. General Appearance.-That of a lively, highly-bred little dog, with dainty appearance, smart, compact carriage, and profuse coat. These dogs should be essentially stylish in movement, lifting the feet high when in motion, carrying the tail (which is 
heavily feathered) proudly curved or plumed over the back. In size they vary considerably, but the smaller they are the better, provided type and quality are not sacrificed. "Wher divided by weight, classes should be for under and over 7 lbs: Coat.-The coat should be long, "prctise, and straight, free from curl or wave, and not be too flat; it should have a tendency to stand out, more particularly at the frill, with profuse feathering on

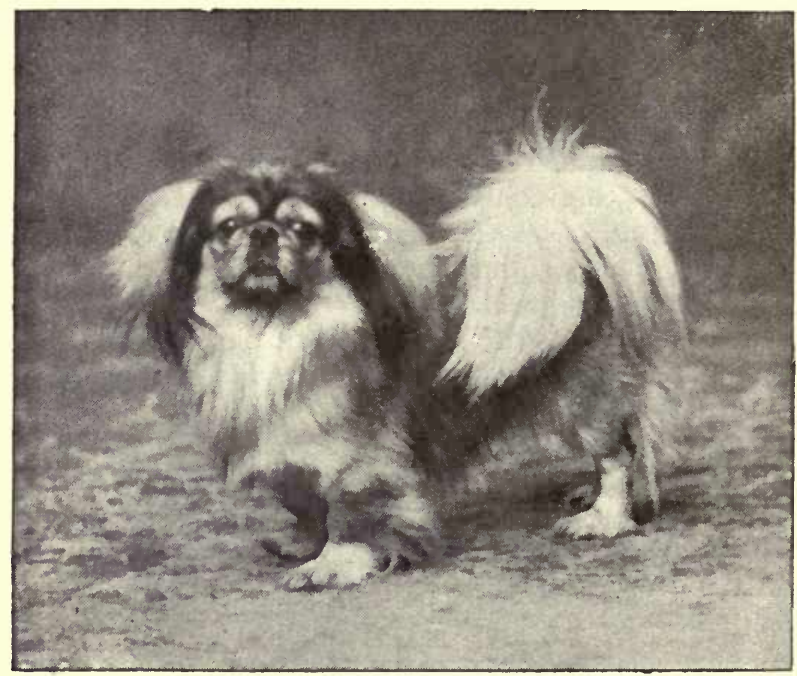

PEKINGESE.

"Yen Chu of Newnham," owned by Mrs. W. H. Herbert.

the tail and thighs. Colour.-The dogs should be either black-and-white or red-and-white-i.e., parti-coloured. The term "red" includes all shades of sable, brindle, lemon, and orange, but the brighter and clearer the red the better. The white should be clear white, and the colour, whether black or red, should be evenly distributed patches over the body, cheek, and ears. Head. -Should be large for size of dog, with a broad skull, 
rounded in front ; eyes large, dark, set far apart ; muzzle very short and wide, and well cushioned-i.e., the upper lips rounded on each side of the nostrils, which should be large and black, except in the case of red-and-white dogs, when a brown-coloured nose is as common as a black one. Ears.- Should be small, set wide apart, and high on the dog's head, and carried slightly forward, V-shaped. Body. - Should be squarely and compactly built, wide in chest, " cobby" in shape. The length of the dog's body should be about its height. Legs and Feet.-The legs should be straight and the bone fine; the feet should be long and hare-shaped. The legs should be well feathered to the feet on the front legs and to the thighs behind. The feet should also be feathered.

The points of Pekingese (as given by the same club). General Appearance.-That of a quaint and intelligent little dog, rather long in body, with heavy front chest, and bow legs-i.e., very much out at elbow-the body falling away lighter behind. The tail should be carried right up in a curve over the animal's back, but not too tightly curled. In size these dogs vary very much, but the smaller the better, provided type and points are not sacrificed. When divided by weight, classes should be for under Io lbs. and over Io lbs. Legs.- Should be short and rather heavy in bone, but not extravagantly so, as coarseness is to be avoided in every point ; they should be well out at elbow, and the feet turned outwards also. Both legs and feet should be feathered. Head.-Should be of medium size, with broad skull, flat between ears, but rounded on the forehead, muzzle very short (not underhung), and very wide. The face should be wrinkled and nostrils black and full. Eyes large and lustrous; ears set high in the head, and Vshaped; they should be moderate in size (the tips never coming below the muzzle), and should be covered with long, silky hair, which extends much below the leather of the ear proper. Colour.-These dogs should either be red, fawn, sable, or brindle, with black masks, face and ear shadings, or else all black. White patches on 
feet or chest, although not a disqualification, should not be encouraged. Coat.-Should be long, flat, and rather silky, except at the frill, where it should stand out, like a lion's mane. The feathering on thighs and tail should be very profuse, and it is preferable that it should be of a lighter colour than the rest of the coat.

There is also the Pekin Palace Dog Association. Secretary, Miss L. C. Smythe, II5, Delaware Mansions, Sutherland Avenue, London, W.

Some other clubs are as follows (but it is in many cases usual to change the Secretary annually, so that these addresses are not all permanent, though letters generally find their mark) :

Halifax and District Yorkshire Terrier Club (Secretary, T. Whiteley, ro, High Street, Halifax).

Manchester and District Yorkshire Terrier Club (Secretary, J. Hardman, 9, Richmond Street, Newton Heath, Manchester).

Oldham Toy Dog Society (Hon. Secretary, A. E. Stansfield, 209, Park Road, Oldham).

Yorkshire Pom Club (Hon. Secretary, E. Poppleton, I, Clarendon Street, Wakefield).

Toy Dog Society of Scotland (Secretary, James Cameron, 6I, Lothian Road, Edinburgh).

North of England Toy Dog Club (Sêcretary, R. Weatherhead, I4, Arctic Parade, Great Horton, Bradford).

Toy Dog Society (Secretary, E. T. Cox, 65 and 66, Chancery Lane, E.C.). 


\section{INDEX}

Abscesses on toes, 46

Amaurosis, 72

Anæmia, 42

Aperients, 56

Appetite, loss of, 48

Areca-nut, 54

Arsenic, 66

Bad doer, the, 5 I

Bare patches, 63

Bat ears, 34

Baths, medicated, 64

Biliousness, 48

Black-and-tan terriers, 37 standard of, 100

Black pugs, 40 standard of, 92

Blenheims, 40 standard of, 86

Bones, 23

Breed, choice of, 30

Breeding, 5

Bronchitis, 74

Bulldogs, toy, 34 standard of, 93

Buying dogs, 4

Canker in ears, 69 in teeth, 45

Caries, dental, 45

Castor oil, 77

Catarrhal distemper, $5^{8}$

Chest diseases, 74

Chill, 48

Clinical thermometer, 48

Clubs, supplementary list, ro4

Coat, 24, 44

Cod liver oil, 44

Cold in eyes, 72

Colds, 73
Conditioning, 72

Coughs, 73

Dew-claws, 73

Digestive tonic, 50

Disagreeable breath, 5 I

Discharge after pupping, I 3

Distemper, 57

Docking, 46

Ears, 69 to alter carriage of, 70

Eczema, 6I, 72

Entering dogs for shows, 27

Epilepsy, 77

Erythema or puppy-pox, 64, 68

Etiquette of shows, 29

Exhibiting, 23

Eyes, 7 I

"Faking," 23

Fatness or obesity, 75

Feeding of Toys, 19, 43, 6 ;

Feet, sore, 72

Fits, 77

French toy bulldog, standard of, 94

Gastritis, 60

Golden ointment, 7 I

Griffons Bruxellois, 37 standard of, 89

Hysteria, 75

Indigestion, 50

Internal parasites, 52

Iron tonic, 44

Italian greyhound, standard of, 97 
Japanese spaniel, 35 standard of, IOI

Kanofelin remedies, 63

Maltese, 4I standard of, 98

Mange, follicular and sarcoptic, 64

Mating bitches, 6

Meat diet, 2I, 43

Mercury, 45, 62

Milk, 22

Missing, 8

Ophthalmia, 7 I

Pekingese spaniels, 35 standard of, IO3

Pityriasis, 63

Poison, 75

Pomeranians, $3 \mathrm{I}$ standard of, 80

Poodles, 27 standard of, 98

Preparing for exhibition, 23

Pugs, 39 standard of, 9I

Puppies, birth of, IO house for, I4 rearing of, I4 size of, 6 skin troubles of, 63 training of, I 8

Pupping, 9
Rashes, 62

Relapse from distemper, 59

Requisites for shows, 28

Ringworm, 67

Round worms, 55

Salt, 76

Scavenging, 20

Schipperkes, 32 standard of, 90

Season, 7

Shivering, 74

Shows, chief, 30

Shyness in ring, 29

Skin diseases, 6I

Stomach coughs, 74

Strychnine, 76

Stuttgart disease, 49, 60

Suckling fits, 77

Sulphur ointment, 63

Tape-worms, 52

Tear channels, 42

Teeth, bad, 45

Teething, I 7

fits, 77

Temperature, to take, 49

Toothache, 46

Toy spaniels, standard of, 83

Washing, 26

Worm medicines, 54

Yorkshire terriers, 38 standard of, 96 


\section{FROM MR. EDWARD ARNOLD'S LIST.}

BY THE SAME AUTHOR.

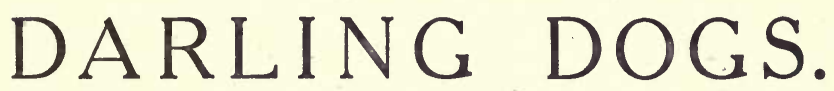

BY

\section{MARGARET LILITH WILLIAMS.}

\section{WITH ILLUSTRATIONS.}

\section{Price 5s. net.}

Times._- An attractive book of talk, light and serious, and of experiences of many kinds, about dogs in the particular and in the abstract by an enthusiast."

GLOBE.- "A delightful volume, especially so to dog-lovers."

WORLD. - " That it is written by one whose heart and soul is in her subject is apparent in the first few pages, and for that very reason they go straight to the heart of every dog-lover. Altogether, a charming volume, excellently illustrated."

DAILY EXPRESS. - "An account of the intimate life of certain dogs, and to those who make dear friends and companions of them it may be confidently recommended."

Evening Standard._- "Practical as well as enthusiastic."

LADY's FIELD.- “ All women who really love dogs-and perhaps all those who love animals of any kind-will like to have 'Darling Dogs,' . . . charmingly illustrated and written from the author's heart."

LONDON : EDWARD ARNOLD, 4I \& 43 MADDOX ST., W. 



\section{UNIVERSITY OF CALIFORNIA LIBRARY}

BERKELEY

Return to desk from which borrowed.

This book is DUE on the last date stamped below.

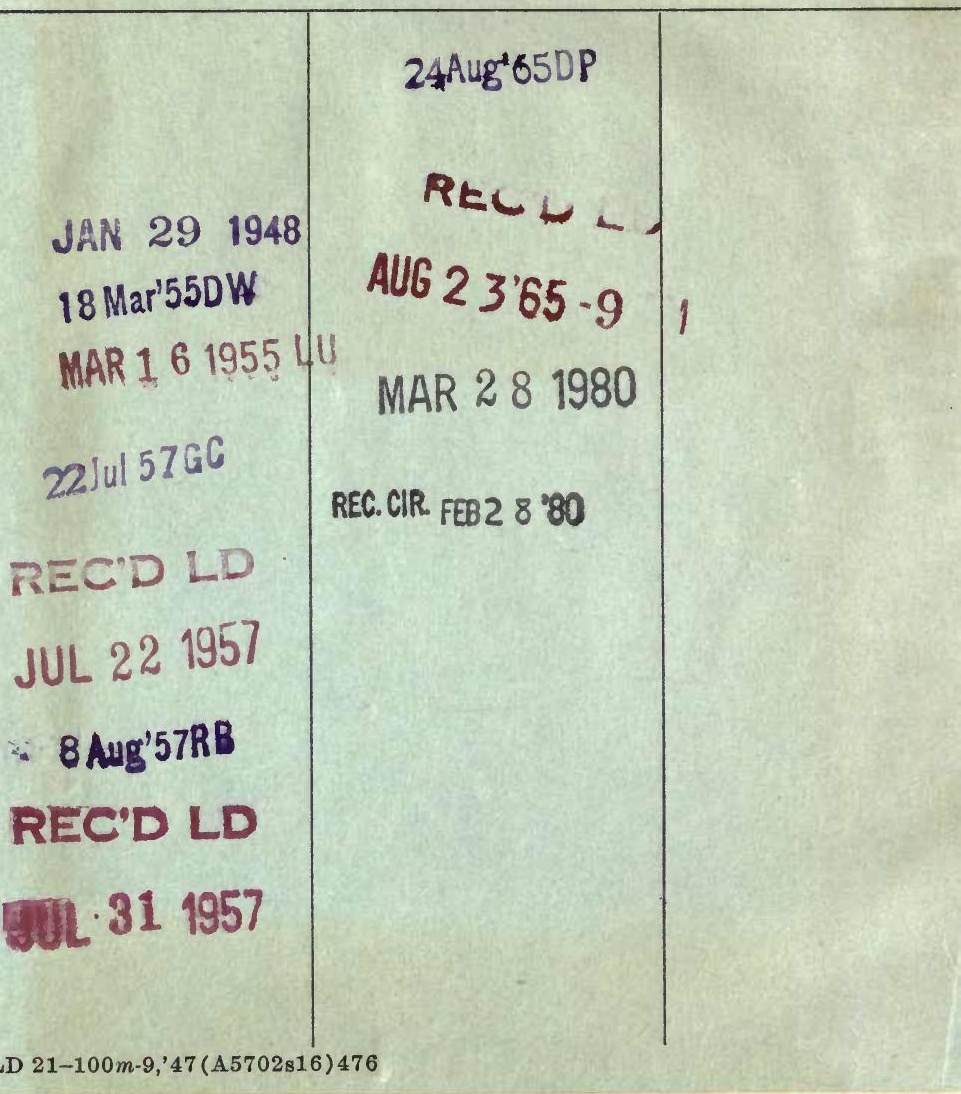




\section{YB $16 / 23$}

4

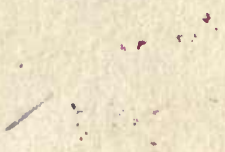

\section{5}

\section{UNIVERSITY OF CALIFORNIA LIBRARY}

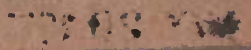


